\title{
SEARCH FOR A COUPLING SCHEME IN HEAVY DEFORMED NUCLEI: THE PSEUDO SU(3) MODEL
}

\author{
R. D. RATNA RAJU ${ }^{\dagger}$, J. P. DRAAYER and K. T. HECHT \\ Department of Physics, The University of Michigan, Ann Arbor, Michigan 48104, USA ${ }^{\dagger \dagger}$
}

Received 30 October 1972

\begin{abstract}
To test the possible usefulness of the pseudo SU(3) coupling scheme a few nuclear properties are examined which can be expected to be governed largely by the $\left(1 g_{\frac{7}{2}} 2 d_{\frac{4}{2}} 2 d_{4} 3 s_{\frac{1}{2}}\right)$ part of the proton configuration for odd- $Z$ nuclei and the $\left(1 \mathrm{~h}_{\frac{2}{2}} 2 \mathrm{f}_{\frac{7}{3}} 2 \mathrm{f}_{\frac{3}{2}} 3 \mathrm{p}_{\frac{1}{2}} 3 \mathrm{p}_{\frac{1}{2}}\right)$ part of the neutron configuration for odd- $N$ nuclei. The equivalence between these configurations and pseudo oscillator shells $\left(\tilde{\mathrm{f}}_{\frac{3}{2}} \tilde{\mathrm{f}}_{\frac{1}{2}} \tilde{\mathrm{p}}_{\frac{2}{2}} \tilde{\mathrm{p}}_{\frac{1}{2}}\right)$ and $\left(\tilde{\mathrm{g}}_{\frac{2}{2}} \tilde{\mathrm{g}}_{\frac{7}{2}} \tilde{\mathrm{d}}_{\frac{1}{2}} \tilde{\mathrm{d}}_{\frac{1}{2}} \tilde{\mathrm{s}}_{\frac{1}{2}}\right)$ is exploited to show that the low-lying natural-parity rotational bands in deformed nuclei can be described approximately by manyparticle states which are coupled to leading pseudo SU(3) representation (maximum possible value of $2 \tilde{\lambda}+\tilde{\mu}$ ) of these configurations. The simple model in which the natural-parity part of the proton and neutron configurations are coupled to leading pseudo SU(3) representation predicts ground state magnetic moments in remarkably good agreement with experiment. The strong hindrance factors observed in certain interband M1 transition probabilities are, however, not predicted by this model. The diagonalization of a simple effective interaction within the leading pseudo SU(3) representation leads to spectra with the experimentally observed ordering of the $K$-bands. (The case of the Eu and Tm isotopes has been examined in some detail since these are expected to have leading pseudo $S U(3)$ representations with the same quantum numbers $(\tilde{\lambda} \tilde{\mu})$ but with quite different ordering of the $K$-bands.)
\end{abstract}

\section{Introduction}

For light nuclei $(A \lesssim 25)$ the rotational features of nuclear spectra can be understood in a many-particle spherical shell-model framework in terms of the SU(3) coupling scheme of Elliott ${ }^{1,2}$ ). For heavier, deformed nuclei, however, the effect of the spin-orbit interaction becomes so important that it alters the closing of major oscillator shells, and hence there is no hope of extending the real SU(3) model to higher regions of the periodic table. In the $3 \mathrm{~s}-2 \mathrm{~d}-1 \mathrm{~g}(N=4)$ shell, for example, the $1 \mathrm{~g}_{\frac{2}{2}}$ member of the $1 g_{\frac{2}{2}}-1 g_{\frac{7}{2}}$ doublet is depressed in energy completely out of the $g_{\frac{1}{4}} d_{\frac{1}{4}} d_{\frac{3}{2}} s_{\frac{1}{2}}$ region (leading to the shell closure at 50 rather than 40 ). The recent assignment of pseudo orbital and pseudo spin angular momenta ${ }^{3-6}$ ) to the single-particle states of the shell model has, on the other hand, led to the possibility of new intermediate coupling schemes which may have applications even in heavy deformed nuclei. In these coupling schemes the positive-parity proton orbits $1 \mathrm{~g}_{\frac{3}{3}} 2 \mathrm{~d}_{\frac{8}{4}} 2 \mathrm{~d}_{\frac{3}{3}} 3 \mathrm{~s}_{\frac{1}{4}}$, being filled

† Present address: Physics Dept., Andhra University, Waltair, AP, India.

t† Work supported by the US National Science Foundation. 
in nuclei with $Z>50$ for example, can be assigned pseudo orbital and spin quantum numbers $\tilde{l}=3, \tilde{l}=1$, and $\tilde{s}=\frac{1}{2}$ and together form a shell $\tilde{\mathrm{f}}_{\frac{3}{2}} \tilde{\mathrm{f}}_{\frac{3}{2}} \tilde{\mathrm{p}}_{\frac{3}{2}} \tilde{\mathrm{p}}_{\frac{1}{2}}$ with pseudo major oscillator shell number $\tilde{N}=3$. The pseudo spin-orbit coupling scheme has proved very useful in the study of 82-neutron nuclei ${ }^{6-8}$ ) where the active protons are filling mainly the $\lg _{\frac{2}{2}} 2 \mathrm{~d}_{\frac{3}{2}}$ single-particle levels, that is, the members of a single pseudo spin-orbit doublet. The validity of the pseudo coupling schemes has also been examined for the Ni region ${ }^{3}$ ) where the equivalence between the real $f_{\frac{3}{2}} p_{\frac{3}{2}} p_{\frac{1}{2}}$ singleparticle levels and a pseudo $\tilde{d}_{\frac{8}{2}} \tilde{d}_{\frac{3}{2}} \tilde{s}_{\frac{1}{2}}$ description has been exploited. In particular, the approximate validity of a pseudo $\mathrm{SU}(3)$ coupling scheme for this pseudo $\tilde{\mathrm{d}} \tilde{\mathrm{s}}$ shell has been established by the recent work of Strottman ${ }^{9}$ ) on the spectra of nuclei with $A=59$ and 60 .

In the heavier deformed nuclei of the rare-earth region the natural-parity active proton orbits $g_{\frac{7}{2}} d_{\frac{3}{2}} d_{\frac{1}{2}} s_{\frac{1}{2}}$ can be considered nearly degenerate, while the $g_{\frac{3}{2}}$ orbit is lost to this major shell and replaced by the unnatural-parity orbit $h_{\frac{4}{2}}$. The experimental evidence shows that there is relatively little excitation out of the filled $g_{\frac{2}{2}}$ level. Spectroscopic factors for single-proton transfer reactions are much less to the $j=\frac{9}{2}$ members of positive-parity rotational bands compared with those for $j \leqq \frac{7}{2}$. For the deformations found in the rare-earth region, the positive-parity Nilsson states rarely have a $\mathrm{g}_{\frac{2}{2}}$ content greater than about $10 \%$. Although a full shell-model description of the proton configurations of such nuclei would have to cope with the negative-parity $1 h_{\sharp}$ orbit, it may be useful to consider the natural-parity part of the proton configuration as made up solely of the pseudo oscillator shell with $\tilde{N}=3,\left(g_{\frac{7}{3}} d_{\frac{3}{4}} d_{\frac{3}{2}} s_{\frac{3}{2}}\right)^{n}=$ $\left(\tilde{\mathrm{f}}_{\frac{3}{2}} \tilde{\mathrm{f}}_{\frac{3}{2}} \tilde{\mathrm{p}}_{\frac{3}{2}} \tilde{\mathrm{p}}_{\frac{1}{2}}\right)^{n}$ and to examine the possibility that the lowest states of such configurations are coupled to the important representations of a pseudo SU(3) group, where the connection between the pseudo $\mathrm{SU}(3)$ representations and the pseudo oscillator quantum numbers parallels that between the real SU(3) model of Elliott and the real oscillator quantum numbers. Similar considerations hold for the natural-parity part of the neutron configurations in the rare-earth region, with $\left(h_{\frac{3}{2}} f_{\frac{7}{2}} f_{\frac{5}{2}} p_{\frac{3}{2}} p_{\frac{1}{2}}\right)^{n}=$ $\left(\tilde{\mathrm{g}}_{\frac{3}{2}} \tilde{\mathrm{g}}_{\frac{1}{2}} \tilde{\mathrm{d}}_{\frac{3}{2}} \tilde{\mathrm{d}}_{\frac{1}{2}} \tilde{\mathrm{s}}_{\frac{1}{2}}\right)^{n}$, and also the natural-parity parts of the proton and neutron configurations in the actinides.

In a strongly deformed nucleus, the real SU(3) coupling scheme can be expected to be applicable in the limit in which (i) the asymptotic oscillator quantum numbers $\left[N n_{z} \Lambda\right]$ for the single-particle states of the deformed field are approximately good quantum numbers, and (ii) the two members of an $\left[\mathrm{Nn}_{z} \Lambda\right]$ Nilsson doublet with $\Omega=\Lambda \pm \frac{1}{2}$ are nearly degenerate. If these two conditions are met, the filling of the single-particle states (subject to the Pauli principle) in the order $n_{z}=N, n_{z}=N-1$, ... will lead to a many-particle state which, following the technique of Elliott ${ }^{1,2}$ ), becomes an intrinsic state of highest weight for the so-called leading representation of $\mathrm{SU}(3)$; that is, the representation $(\lambda \mu)$ with the largest possible value of $2 \lambda+\mu$ and, for fixed value of this sum, the largest possible value of $\mu$. For nuclei in the first half of the $2 \mathrm{~s}-1 \mathrm{~d}$ shell these two conditions are met to a sufficient degree of approximation. Detailed shell-model calculations have shown that the ground state rotational bands 


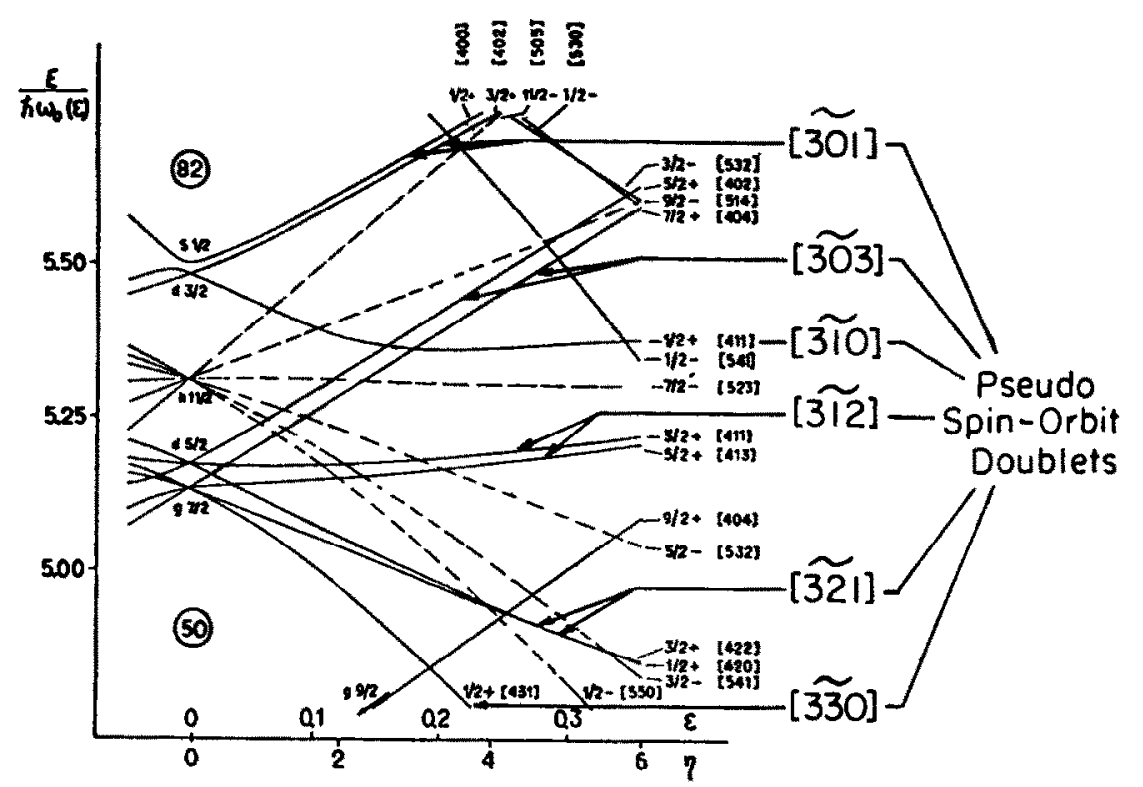

Fig. 1. The Nilsson diagram, $50 \leqq Z \leqq 82$, labelled with pseudo oscillator quantum numbers $\left[\tilde{N} \tilde{n}_{z} \tilde{A}\right]$. (The diagram is adapted from ref. ${ }^{11}$ ).)

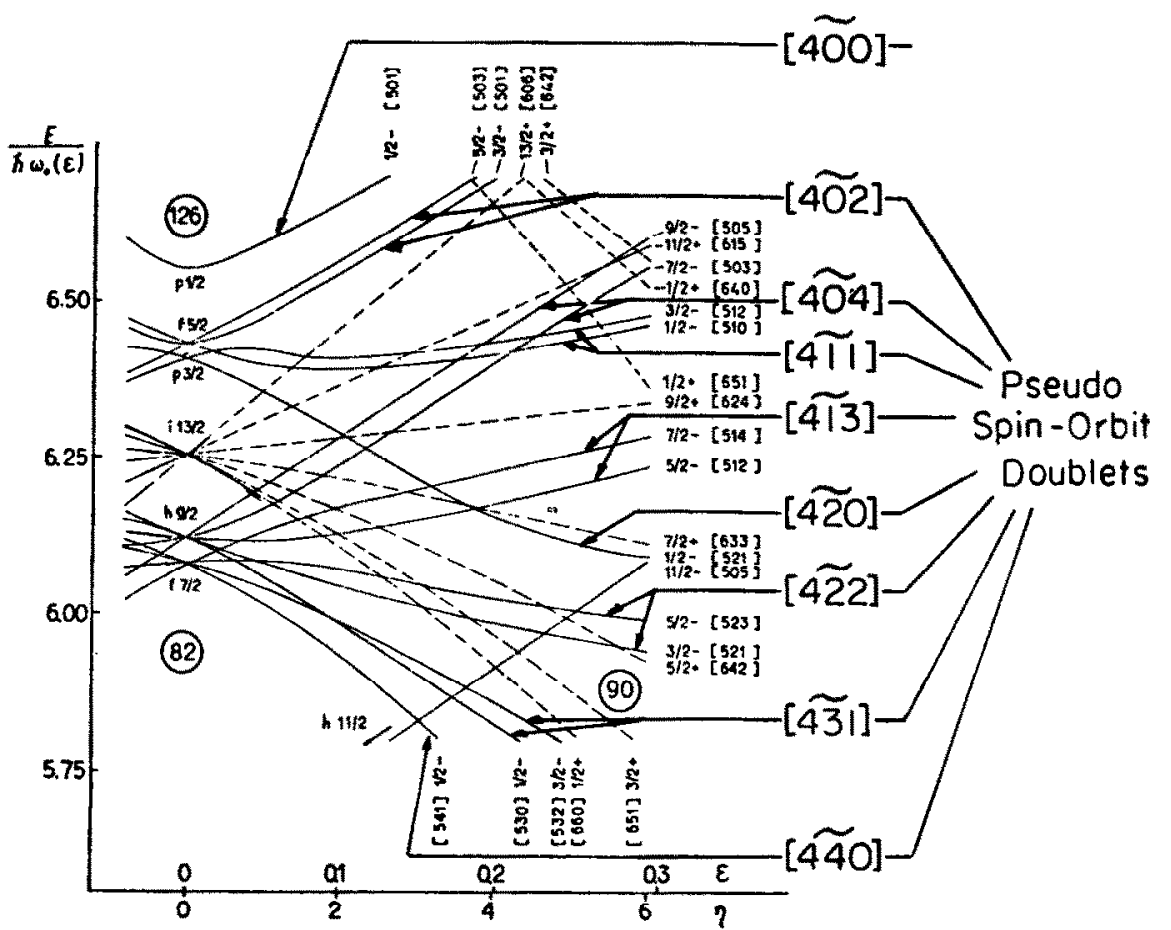

Fig. 2. The Nilsson diagram, $82 \leqq N \leqq 126$, labelled with pseudo oscillator quantum numbers $\left[\tilde{N} \tilde{n}_{z} \tilde{\Lambda}\right]$. (The diagram is adapted from ref. ${ }^{11}$ ).) 
in these nuclei have eigenvectors which are composed predominantly of the leading $\mathrm{SU}(3)$ representations.

However, in the rare-earth nuclei, although the deformations are large, the spinorbit splittings are also very large, so that Nilsson levels $\left[N n_{z} \Lambda\right]$ with $\Omega=\Lambda \pm \frac{1}{2}$ are split by large amounts. The Nilsson diagrams for the $N=4$ (proton) and $N=5$ (neutron) shells of the rare-earth region are shown in figs. 1 and 2 . The proton levels [404] with $\Omega^{\pi}=\frac{7}{2}^{+}$and $\frac{9}{2}^{+}$, for example, are split by an amount which is of the order of the major shell separation $\hbar \omega_{0}$ throughout the range of deformations characteristic of the rare-earth region. In the deformed force field the levels which grow out of the spherical shell-model states of the $\mathrm{g}_{\frac{7}{2}} \mathrm{~d}_{\frac{3}{2}} \mathrm{~d}_{\frac{3}{2}} \mathrm{~s}_{\frac{1}{2}}$ or pseudo $\tilde{\mathrm{f}}_{\frac{3}{2}} \tilde{\mathrm{f}}_{\frac{3}{2}} \tilde{\mathrm{p}}_{\frac{3}{2}} \tilde{\mathrm{p}}_{\frac{1}{2}}$ shell can also be assigned pseudo harmonic oscillator quantum numbers $\left[\tilde{N} \tilde{n}_{z} \tilde{\Lambda}\right]$ with $\bar{N}=3$. These pseudo oscillator quantum numbers are shown for the proton states in fig. 1 , and for the neutron states $(\tilde{N}=4)$ in fig. 2 . For example, the Nilsson states $\left.[411]]^{\frac{3}{2}}{ }^{+},[413]\right]^{\frac{5}{2}}{ }^{+}$ become the members of a pseudo spin-orbit doublet $\left[\tilde{N} \tilde{n}_{z} \tilde{\Lambda}\right]=[\tilde{3} \tilde{1} \tilde{2}]$ with $\Omega=\tilde{\Lambda} \pm \frac{1}{2}$. Note in particular that pseudo spin-orbil doublets of the type $[\tilde{3} \tilde{1} \tilde{2}] \frac{5}{2}^{+},{ }^{\frac{3}{2}}{ }^{+},[\tilde{3} \tilde{0} \tilde{3}]_{\frac{7}{2}}^{+}$, $\frac{5}{2}^{+}, \ldots$ are almost degenerate. Moreover the asymptotic limit $\left[\tilde{N} \tilde{n}_{z} \tilde{\Lambda}\right]$ may be expected to be approached more rapidly than the corresponding asymptotic limit $\left[N n_{z} \Lambda\right]$ since the coefficients of terms of the type $\tilde{\boldsymbol{l}} \cdot \tilde{\boldsymbol{s}}$ and $\tilde{\boldsymbol{l}}^{2}$, needed to reproduce the single-particle spectrum in the spherical limit, are smaller than the coefficients of the corresponding $\boldsymbol{l} \cdot \boldsymbol{s}$ and $\boldsymbol{s}^{2}$ terms of the Nilsson Hamiltonian. For deformations characteristic of the rare-earth region we might expect the quantum numbers $\left[\tilde{N} \tilde{n}_{z} \tilde{\Lambda}\right]$ to be better than the quantum numbers $\left[N n_{z} \Lambda\right]$, provided the $g_{\frac{g}{2}}$ content of the states is indeed negligible. This is borne out by some of the simple properties of the Nilsson states. For example, the decoupling parameters, $a$, for $K=\frac{1}{2}$ rotational bands have the value $a=(-1)^{N} \delta_{A 0}$ in the asymptotic limit in which the real Nilsson quantum numbers $\left[\mathrm{Nn}_{z} \Lambda\right]$ are rigorously good quantum numbers. On the other hand, in the asymptotic limit in which the pseudo oscillator numbers $\left[\tilde{N} \tilde{n}_{z} \tilde{\Lambda}\right]$ are good quantum numbers, the decoupling parameters would have the values $a=(-1)^{\tilde{N}} \delta_{\tilde{A} 0}$ (see the appendix). Tables 1 and 2, taken from the recent compilation of Bunker and Reich ${ }^{10}$ ) show a list of all the experimentally determined decoupling parameters for $K=\frac{1}{2}$ bands of the rare-earth region. (Values enclosed in parentheses may be based on tentative assignments or subject to strong interband Coriolis mixing. Also, $K=\frac{1}{2}$ bands which are known to have significant $\gamma$-vibrational admixtures have been omitted from the list.) In the odd- $Z$ nuclei, e.g., the $K=\frac{1}{2}$ rotational bands based on the Nilsson state $[411] \frac{1}{2}^{+}$, which corresponds to pseudo oscillator quantum numbers $[\tilde{3} \tilde{1} \tilde{0}]$, have decoupling parameters closer to the value $a=-1$, the asymptotic limit for the $\left[\tilde{N} \tilde{n}_{z} \tilde{\Lambda}\right]$ scheme. In the odd $-N$ nuclei (table 2 ), similarly, the experimentally observed decoupling parameters are invariably closer to the asymptotic limiting values for the $\left[\tilde{N} \tilde{n}_{z} \tilde{\Lambda}\right]$ scheme. Other simple properties can be calculated for both asymptotic limits to compare their approximate validity. Tables 3 and 4, for example, show the magnetic moments of the natural-parity Nilsson states for the odd- $Z$ and odd- $N$ nuclei of the rare-earth region in the Bohr-Mottelson-Nilsson rotational model (for rotational 
TABLE 1

Decoupling parameters $a$ for odd $-Z$ nuclei

\begin{tabular}{|c|c|c|}
\hline $\begin{array}{l}{\left[N n_{z} \Lambda\right]} \\
{\left[\tilde{N} \tilde{n}_{z} \tilde{\Lambda}\right]}\end{array}$ & Nucleide & $\left.a_{\exp }{ }^{2}\right)$ \\
\hline $\begin{array}{l}{[411]} \\
{[\tilde{3} \tilde{1} \tilde{0}]}\end{array}$ & 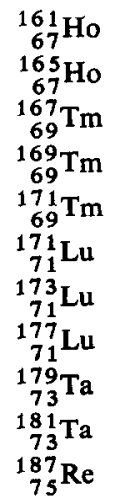 & $\begin{array}{c}-0.75 \\
-0.44 \\
-0.72 \\
-0.77 \\
-0.86 \\
-0.71(-1.2) \\
(-0.75) \\
-0.91 \\
(\approx-0.8) \\
(-0.9) \\
(-1.13)\end{array}$ \\
\hline
\end{tabular}

Asymptotic limits for all entries in this table are:

$a=0$ for $\operatorname{good}\left[N n_{2} \Lambda\right]$

$a=-1$ for $\operatorname{good}\left[\tilde{N} \tilde{n}_{z} \tilde{\Lambda}\right]$

") Taken from ref. ${ }^{10}$ ).

TABLE 2

Decoupling parameters $a$ for odd- $N$ nuclei

\begin{tabular}{|c|c|c|c|c|c|}
\hline $\begin{array}{l}{\left[N n_{z} \Lambda\right]} \\
{\left[\tilde{N n_{z}} \tilde{\Lambda}\right]}\end{array}$ & Nucleide & $\left.a_{\exp }{ }^{a}\right)$ & $\begin{array}{l}{\left[N n_{z} \Lambda\right]} \\
{\left[\tilde{N} \tilde{n}_{z} \tilde{\Lambda}\right]}\end{array}$ & Nucleide & $\left.a_{\exp }{ }^{a}\right)$ \\
\hline [521] & ${ }^{165} \mathrm{Dy}_{99}$ & 0.58 & [530] & ${ }^{135} \mathrm{Gd}_{91}$ & 0.12 \\
\hline \multirow[t]{14}{*}[\tilde{4}\tilde{2}\tilde{0}]{} & ${ }^{167} \mathrm{Er}_{99}$ & 0.70 & {$[\tilde{4} \tilde{3} \tilde{1}]$} & & \\
\hline & ${ }^{169} \mathrm{Yb}_{99}$ & 0.79 & [510] & ${ }^{173} \mathrm{Yb}_{103}$ & 0.20 \\
\hline & ${ }^{169} \mathrm{Er}_{101}$ & 0.83 & [थี $\tilde{1} \tilde{1}]$ & ${ }^{175} \mathrm{Yb}_{105}$ & 0.19 \\
\hline & ${ }^{171} \mathrm{Yb}_{101}$ & 0.85 & & ${ }^{177} \mathrm{Yb}_{107}$ & 0.24 \\
\hline & ${ }^{173} \mathrm{Hf}_{101}$ & 0.82 & & ${ }^{179} \mathrm{Hf}_{107}$ & 0.16 \\
\hline & ${ }^{171} \mathrm{Er}_{103}$ & 0.62 & & ${ }^{181} \mathrm{Hf}_{109}$ & 0.20 \\
\hline & ${ }^{173} \mathrm{Yb}_{103}$ & 0.73 & & ${ }^{183} \mathrm{~W}_{109}$ & 0.19 \\
\hline & ${ }^{175} \mathrm{Hf}_{103}$ & 0.75 & & ${ }^{185} \mathrm{Os}_{109}$ & 0.02 \\
\hline & ${ }^{175} \mathrm{Yb}_{105}$ & 0.75 & & ${ }^{187} \mathrm{Os}_{111}$ & 0.05 \\
\hline & ${ }^{179} W_{105}$ & 0.82 & & ${ }^{187} W_{113}$ & 0.0 \\
\hline & ${ }^{179} \mathrm{Hf}_{107}$ & 0.67 & & $189 \mathrm{Os}_{113}$ & -0.17 \\
\hline & ${ }^{181} W_{107}$ & 0.48 & \multirow{2}{*}{\multicolumn{2}{|c|}{$\begin{array}{l}\text { Asymptotic limits: } \\
\qquad a=-1 \text { for good }\left[N n_{z} \Lambda\right]\end{array}$}} & \\
\hline & ${ }^{183} \mathrm{~W}_{109}$ & 0.7 & & & \\
\hline & ${ }^{185} \mathrm{~W}_{111}$ & 0.86 & $a=\mathrm{c}$ & $\operatorname{ood}\left[\tilde{N} \tilde{n}_{z} \tilde{\Lambda}\right]$ & \\
\hline
\end{tabular}

Asymptotic limits:

$a=0$ for good $\left[\mathrm{Nn}_{z} \Lambda\right]$

$a=+1$ for $\operatorname{good}\left[\tilde{N} \tilde{n}_{z} \tilde{\Lambda}\right]$

a) Taken from ref. ${ }^{10}$ ). 
ground states $I=K=\Omega$ ) in the two limiting cases in which either the asymptotic quantum numbers $\left[N n_{z} \Lambda\right]$ or $\left[\tilde{N} \tilde{n}_{z} \hat{\Lambda}\right]$ are assumed to be good quantum numbers for the last (odd) nucleon. (The derivation for the pseudo oscillator limit is given in the appendix; the $\mu$-values have been calculated with free nucleon $g_{s}$ and $g_{l}$ values and approximate average values of $y_{R}, g_{R}=0.4$ for the $N=4,(\tilde{N}=3)$ odd-proton case, and $g_{R}=0.3$ for the $N=5(\tilde{N}=4)$ odd-neutron case.) Tables 3 and 4 also list the experimentally observed magnetic moments for the natural-parity ground states of the rare-earth nuclei. (To save space the experimental values are not identified with the names of the nucleides, but these can be read off from tables 9 and 10.) Although the agreement between the predicted asymptotic numbers and the experimental values is not striking for either limiting case, the results for the asymptotic limit $\left[\tilde{N} \tilde{n}_{z} \hat{\Lambda}\right]$, using pseudo oscillator quantum numbers, are in general in much better overall agreement with the experimental values. (Calculations for the actual deformation parameters of the rare-earth nuclei, using the exact Nilsson functions, show that the deviations may result from the shortcomings of the rotational model as much as from deviations from the asymptotic limit.)

The near degeneracy of the pseudo spin-orbit doublets $\left[\tilde{N} \tilde{n}_{z} \hat{\Lambda}\right]$ with $\Omega=\hat{A} \pm \frac{1}{2}$, and the approximate validity of the asymptotic quantum numbers $\left[\tilde{N} \tilde{n}_{z} \hat{\Lambda}\right]$ thus suggests that a pseudo SU(3) coupling scheme may be a good approximation for the many-particle states of these configurations. The filling of the deformed-field single-

TABLE 3

Asymptotic limits for magnetic moments (odd- $Z$ nuclei)

\begin{tabular}{|c|c|c|c|c|}
\hline$\left[N n_{z} A\right]$ & {$\left[\tilde{N} \tilde{n}_{z} \tilde{A}\right] \Omega^{n}$} & $\begin{array}{c}\text { Good } \\
{\left[N n_{z} \Lambda\right]}\end{array}$ & $\begin{array}{c}\text { Good } \\
{\left[\tilde{N} \tilde{n}_{z} \tilde{\Lambda}\right]}\end{array}$ & Exp. \\
\hline$[400]$ & {$[\tilde{30} \tilde{0} \tilde{1}]^{+}$} & 2.79 & 2.34 & \\
\hline$[402]$ & {$[\tilde{3} \tilde{0} \tilde{1}]^{3}{ }^{+}$} & -0.24 & -0.01 & $\left\{\begin{array}{l}0.145 \\
0.158\end{array}\right.$ \\
\hline$[402]$ & {$[\tilde{30} \tilde{3}]^{3}+$} & 3.71 & 3.35 & $\begin{array}{l}\left\{\begin{array}{l}3.172 \\
3.204\end{array}\right. \\
2.23\end{array}$ \\
\hline [404] & $[\tilde{3} 0 \tilde{3}]]_{2}^{z+}$ & 1.25 & 1.65 & $\left\{\begin{array}{l}2.24 \\
2.36\end{array}\right.$ \\
\hline [411] & $[\tilde{3} \tilde{1} \tilde{0}]]^{+}$ & -0.46 & 0.22 & $\left\{\begin{array}{l} \pm 0.229 \\
\perp 0.08\end{array}\right.$ \\
\hline [411] & $[\tilde{3} \tilde{1} \tilde{2}]]_{2}^{+}$ & 2.52 & 1.60 & $\left\{\begin{array}{l} \pm 2.0 \\
+1.99\end{array}\right.$ \\
\hline [413] & {$[\tilde{312}]^{5}{ }^{+}$} & 0.43 & 1.53 & 1.53 \\
\hline$[420]$ & $[\tilde{3} \tilde{2} \tilde{1}]]^{+}$ & 2.79 & 0.81 & \\
\hline$[422]$ & $[\tilde{3} \tilde{2} \tilde{1}]]^{+}$ & -0.24 & 1.37 & \\
\hline [431] & {$[\tilde{330}]_{\frac{1}{2}}+$} & -0.46 & 1.85 & \\
\hline
\end{tabular}


TABLE 4

Asymptotic limits for magnetic moments (odd- $N$ nuclei)

\begin{tabular}{|c|c|c|c|c|}
\hline$\left[\mathrm{Nn}_{z}, \Lambda\right]$ & {$\left[\tilde{N} \tilde{n}_{z} \tilde{\Lambda}\right] \Omega^{\pi}$} & $\begin{array}{l}\text { Good } \\
{\left[N n_{z} \Lambda\right]}\end{array}$ & $\begin{array}{l}\text { Good } \\
{\left[\tilde{N} \tilde{n}_{z} \tilde{\Lambda}\right]}\end{array}$ & Exp. \\
\hline [501] & {$[\tilde{400}]_{\frac{1}{2}}^{-}$} & 0.74 & 0.64 & \\
\hline [503] & $[\tilde{4} \tilde{0} \tilde{2}]]_{2}^{-}$ & 1.58 & 1.40 & \\
\hline [501] & $[\overline{402}]]_{2}^{3}-$ & -0.97 & -0.82 & \\
\hline [505] & {$[\tilde{4} \tilde{0} \tilde{4}] g^{-}$} & 1.81 & 1.53 & \\
\hline [503] & $\tilde{[404}]_{\frac{7}{2}}^{-}$ & -1.25 & -0.98 & \\
\hline [512] & {$[\tilde{4} \tilde{1} \tilde{1}]^{3}-$} & 1.33 & 0.89 & 0.6566 \\
\hline$[510]$ & {$[\tilde{4} \tilde{1} \tilde{1}]_{\frac{1}{2}}^{-}$} & 0.84 & 0.70 & 0.117 \\
\hline [514] & {$[\tilde{4} \tilde{1} \tilde{3}]_{\frac{2}{2}}^{-}$} & 1.72 & 0.91 & 0.61 \\
\hline$[512]$ & {$[\tilde{4} \tilde{1} \tilde{3}]_{\frac{5}{2}}^{-}$} & -1.15 & -0.41 & \\
\hline [521] & {$[\tilde{4} \tilde{20}]_{\frac{1}{2}}^{-}$} & 0.74 & 0.64 & $\left\{\begin{array}{l}0.513 \\
0.4919\end{array}\right.$ \\
\hline [523] & {$[\tilde{4} \tilde{2} \tilde{2}]^{5}{ }^{-}$} & 1.58 & 0.27 & $\left\{\begin{array}{c}0.64 \\
\pm 0.65 \\
1.1 \\
0.55\end{array}\right.$ \\
\hline [521] & $[\tilde{4} \tilde{2} \tilde{2}]]^{3}-$ & -0.97 & 0.14 & $\left\{\begin{array}{l}-0.254 \\
-0.339\end{array}\right.$ \\
\hline [532] & {$[\tilde{\mathbf{4}} \tilde{3} \tilde{1}]^{\underline{a}}{ }^{-}$} & 1.33 & -0.17 & \\
\hline [530] & $[\tilde{4} \tilde{3} \tilde{1}]]_{2}^{-}$ & 0.84 & 0.69 & \\
\hline [541] & {$[\tilde{4} \tilde{4} \tilde{4}]_{\frac{1}{2}}^{-}$} & 0.74 & 0.64 & \\
\hline
\end{tabular}

particle states $\left[\tilde{N} \tilde{n}_{z} \tilde{\Lambda}\right]$ in the order $\tilde{n}_{z}=\tilde{N}, \tilde{n}_{z}=\tilde{N}-1, \ldots$ will lead to a many-particle state which, again following the discussion of Elliott, becomes an intrinsic state of highest weight for the leading pseudo $S U(3)$ representation with maximum possible value of $2 \tilde{\lambda}+\tilde{\mu}$ and, for fixed value of this sum, maximum possible value of $\tilde{\mu}$. Since the active protons and neutrons are filling different major shells $(\tilde{N})$ in the heavy deformed nuclei, the many-particle states would also have good spatial (and hence spin) symmetry in the separate pseudo spaces of the natural-parity proton and neutron configurations. The classification of the many-particle states according to pseudo SU(3) symmetry and total pseudo spin $\widetilde{S}$ may therefore be expected to be physically meaningful, and the low-lying rotational bands of heavy deformed nuclei may be expected to have eigenvectors with major components of the natural-parity parts of the configuration given by the leading pseudo $S U(3)$ representation, (maximum value of $2 \tilde{\lambda}+\tilde{\mu})$ and lowest possible pseudo spin, $\tilde{S}=0$ for even and $\tilde{S}=\frac{1}{2}$ for odd proton (or neutron) numbers.

Unfortunately, the classification of the many-particle states is complicated by the presence of the unnatural-parity single-particle level in the midst of the natural-parity 
levels, for example the $1 h_{\frac{14}{2}}$ level in the $g_{\frac{3}{2}} d_{\frac{1}{2}} d_{\frac{3}{2}} s_{\frac{1}{2}}$ proton shell. However, insofar as the properties of the low-lying rotational bands in an odd- $Z$ nucleus, for example, are governed predominantly by a single configuration $\left(\mathrm{g}_{\frac{7}{2}} \mathrm{~d}_{\frac{5}{2}} \mathrm{~d}_{\frac{3}{3}} \mathrm{~s}_{\frac{1}{2}}\right)^{n}\left(\mathrm{~h}_{\frac{1}{2}}\right)_{J=0}^{\mathrm{Z}-50-n}$, with fixed odd $n$, the properties of these bands may perhaps be related to the properties of the leading pseudo SU(3) representations. These considerations limit the kinds of nuclear properties which can be studied in terms of the pseudo SU(3) scheme. Accurate predictions of energy spacings, for example, would surely be influenced by a small amount of mixing between the above proton configuration and configurations of the type $\left(g_{\frac{7}{2}} d_{\frac{3}{2}} d_{\frac{3}{2}} s_{\frac{1}{2}}\right)^{n \pm 2}\left(h_{\frac{1}{2}}\right)^{Z-50-n \mp 2}$, not to mention the interactions between the oddproton configurations with the neutron configurations. However, we might expect that the magnetic moments of positive-parity ground states of odd- $Z$ nuclei in the rareearth region, for example, might be understood in terms of the leading pseudo SU(3) representations of the configurations $\left(g_{\frac{3}{2}} \mathrm{~d}_{\frac{3}{3}} \mathrm{~d}_{\frac{3}{2}} \mathrm{~s}_{\frac{1}{2}}\right)^{n}$, with odd $n$. To determine $n$ for a particular nucleus we shall use the known systematics of the region based on the Nilsson scheme and the experimental observations for the rare-earth nuclei. For example, for the ${ }_{63}$ Eu isotopes, with 13 protons outside the closed shell with $Z>50$, the Nilsson diagram (fig. 1) would lead us to expect that $n=7$. (With a positive deformation, $\varepsilon \approx 0.3$, we would expect to fill the three positive-parity levels $[\tilde{3} \tilde{3} \tilde{0}] \frac{1}{2}^{+}$, $[\tilde{3} \tilde{2} \tilde{1}]_{2}{ }^{+},[\tilde{3} \tilde{2} \tilde{1}]_{2}^{1^{+}}$with six protons and the three lowest negative-parity levels growing out of the $1 \mathrm{~h}_{\frac{1}{2}}$ state also with six protons, leaving the 13 th or 7 th positive-parity proton for the positive-parity level [ $\tilde{3} \tilde{1} \tilde{2}$ ] with $\Omega^{\pi}=\frac{5}{2}^{+}$or $\frac{3}{2}^{+}$.) This analysis also fixes the $\tilde{\lambda}$ and $\tilde{\mu}$ values for the leading pseudo $\mathrm{SU}(3)$ representation for $n=7$. The maximum value of $2 \tilde{\lambda}+\tilde{\mu}$ is given by the largest possible value of $3 \sum \tilde{n}_{z}-\sum \tilde{N},(=24$ in this case), where $\sum \tilde{n}_{z}$ and $\sum \tilde{N}$ are the sums of the single-particle quantum numbers of the filled pseudo oscillator states. Similarly, $\tilde{\mu}$ for the leading representation is given by the maximum value of $\sum \tilde{n}_{x}-\sum \tilde{n}_{y}$, subject to the above restriction on $\sum \tilde{n}_{z}$. For $n=7$, therefore, the leading pseudo $\mathrm{SU}(3)$ representation is $(\tilde{\lambda} \tilde{\mu})=(11,2)$.

The leading pseudo $\mathrm{SU}(3)$ representations for all odd-proton numbers for nuclei with $Z>50$ are shown in table 5. (The companion table for odd-neutron numbers and $N>82$ can be found in ref. ${ }^{6}$ ).) In cases where more than one pseudo SU(3) representation $(\tilde{\lambda} \tilde{\mu})$ with maximum value for $2 \tilde{\lambda}+\tilde{\mu}$ is possible all such representations are listed since they may all be expected to be important pieces in the wave functions

TABLE 5

Leading pseudo SU(3) representations for odd proton number $50 \leqq Z \leqq 82$

\begin{tabular}{|c|c|c|c|c|c|c|c|c|c|c|}
\hline $\begin{array}{l}\text { Number of odd + parity } \\
\text { protons }\end{array}$ & 1 & 3 & 5 & 7 & 9 & 11 & 13 & 15 & 17 & 19 \\
\hline $\begin{array}{l}\text { Leading } \\
\text { pseudo } \\
\text { SU(3) rep. } \\
(\tilde{\lambda} \tilde{\mu})\end{array}$ & (30) & (71) & $(10,1)$ & $(11,2)$ & $\begin{array}{l}(10,4) \\
(11,2)\end{array}$ & $(11,2)$ & $(9,3)$ & $\begin{array}{l}(47) \\
(55) \\
(63) \\
(71)\end{array}$ & (17) & (03) \\
\hline Possible $\hat{K}$-values & 0 & 1 & 1 & 2,0 & $4,2,0$ & 2,0 & 3,1 & many & 1 & 0 \\
\hline Expected ground state $\tilde{K}$ & $\mathbf{0}$ & 1 & 1 & 2 & 2 & 0 & 3 & 3 & 1 & $\mathbf{0}$ \\
\hline
\end{tabular}


of the low-lying rotational bands. The possible $\tilde{K}$ values (that is $\tilde{K}_{L}$ values) given by the rule $\tilde{R}=\tilde{\mu}, \tilde{\mu}-2, \ldots$ are also shown, and the table also shows the $\tilde{R}$ values expected to be the most likely candidates for the ground state. These are again determined from the systematics of the region with the aid of the Nilsson diagram. In the ${ }_{63} \mathrm{Eu}$ isotopes, for example, the 7th (last) positive-parity proton is expected to occupy the [ $\tilde{3} \tilde{1} \tilde{2}$ ] doublet, leading to the $R$ value, $\widetilde{K}=\hat{A}=2$, corresponding to the observed rotational bands in ${ }_{63}^{153} \mathrm{Eu}$ and ${ }_{63}^{155} \mathrm{Eu}$ with $K_{J}$ of $\frac{5}{2}$ and $\frac{3}{2}$. It is interesting to note that the leading representations contain the expected $\widetilde{K}$ values in every case, although it is not clear, for example, why $\widetilde{K}=2$ should lie lowest for $n=7$ with leading representation $(11,2)$, while for $n=11$, which also has $(11,2)$ as its leading representation, it is $\widetilde{K}=0$ which is expected to lie lowest. This point is examined in sect. 4 .

It is the purpose of this investigation to test in some detail the possible usefulness of the pseudo $\mathrm{SU}(3)$ coupling scheme for nuclei in the rare-earth region. For this purpose, however, we shall have to focus attention on those nuclear properties which are governed mainly by that part of the nucleon configuration corresponding to an odd number of protons in the positive-parity levels or an odd number of neutrons in the negative-parity levels. We might expect the magnetic moments of the natural-parity states to be such a property. They have been calculated in terms of the configurations $\left(\mathrm{g}_{\frac{7}{2}} \mathrm{~d}_{\frac{3}{3}} \mathrm{~d}_{\frac{3}{2}} \mathrm{~s}_{\frac{1}{2}}\right)^{n}$ coupled to leading $(\lambda \tilde{\mu})$ for odd- $Z$ nuclei, and configurations $\left(\mathrm{h}_{\frac{3}{3}} \mathrm{f}_{\frac{2}{2}} \mathrm{f}_{\frac{3}{2}}\right.$ $\left.\mathrm{p}_{\frac{2}{2}} \mathrm{p}_{\hat{t}}\right)^{n}$, again coupled to leading $(\tilde{\lambda} \tilde{\mu})$ for the odd- $N$ nuclei. The results are presented in sect. 3. Since the magnetic moment results should be closely related to the MI transition probabilities, M1 transition probabilities between states of the leading pseudo SU(3) representations have also been calculated. These are discussed in connection with the spectra in sect. 4 . Although precise energy calculations are impossible (the configuration mixing problem for the full proton and neutron configuration would clearly be prohibitive), we might hope that the diagonalization of a good effective interaction within the states of the leading pseudo SU(3) representation of the natural-parity part of the proton (or neutron) configuration (a highly truncated part of the full shell-model space), would at least predict the correct order of the $\widetilde{\mathbf{K}}$ bands. For this reason energy calculations have been carried out for the case $n=7$ (seven active positive-parity protons), which can be expected to apply to the spectra of the ${ }_{63}$ Eu isotopes, and the case $n=11$, which can be expected to apply to the spectra of the ${ }_{11} \mathrm{Tm}$ isotopes. Both of these cases have leading pseudo $\mathrm{SU}(3)$ representation $(11,2)$, but it remains to show that the $\widetilde{K}_{L}=2$ band, split into $K_{J}=\frac{5}{2}$ and $\frac{3}{2}$ bands, is the predicted ground state band for the ${ }_{63} \mathrm{Eu}$ isotopes, while the $\widetilde{R}_{L}=0$ band, with $K_{J}=\frac{1}{2}$, is the predicted ground state band for the ${ }_{69} \mathrm{Tm}$ isotopes, $(n=11)$. The energy calculations for these cases are presented in sect. 4 . Some aspects of the method of calculation are discussed in sect. 2; full details can be found in ref. ${ }^{12}$ ).

\section{Method of calculation}

Thanks to the availability of programs for calculating SU(3) Wigner and Racah coefficients ${ }^{13,14}$ ), the most efficient method of calculation will make use of standard 
Racah fractional parentage techniques and the SU(3) irreducible tensor formalism.

The physically interesting one- and two-body operators are expressed in terms of single-particle creation and annihilation operators for either protons or neutrons: $a_{i \eta 0) l m m_{s}}^{+}, a_{(\eta 0) l m m_{s}}$. Here we use the single-particle $\operatorname{SU}(3)$ label $(\lambda \mu)=(\eta 0)$ in place of the principal quantum number. In most of our applications the quantum numbers will refer to the pseudo coupling schemes, (that is, $\left.(\tilde{\eta} \tilde{0}) \tilde{l} \tilde{m} \tilde{s} \tilde{m}_{s}\right)$. For example, the operator which creates a proton in the magnetic substate $m_{j}$ of the $1 g_{\frac{3}{2}}$ shell is given by

$$
\sum_{\tilde{m} \tilde{m}_{s}}\left\langle\tilde{3} \tilde{m} \tilde{\frac{1}{2}} \tilde{m}_{s} \mid \frac{7}{2} m_{j}\right\rangle a_{(\tilde{3} 0)}^{\dagger} \tilde{3}_{\tilde{m}_{m} \tilde{m}_{s}} \text {. }
$$

But, unless specifically needed, the superscripts ( ${ }^{\sim}$ ) will be omitted in the general discussion, since the results apply to both the real and the pseudo coupling schemes. The single-particle creation operators have SU(3) irreducible tensor character ( $\eta 0)$, while the single-particle annihilation operators transform according to the conjugate representation $(0 \eta)$. Specifically, the irreducible tensor properties are given by

$$
\begin{gathered}
a_{(\eta 0) l m m_{s}}^{\dagger}=t^{(\eta 0) l \frac{1}{m m_{s}}}, \\
(-1)^{\eta+l-m+\frac{1}{2}-m_{s}} a_{(\eta 0) l m m_{s}}=t^{(0 \eta) l}-m_{-}^{\frac{1}{2}}-m_{s} .
\end{gathered}
$$

One-body operators of definite spherical tensor rank are constructed in the usual way

$$
\begin{aligned}
T\left(l^{\prime}, l\right)^{L_{0} S_{0} J_{0}}=\sum_{M_{0}=}\left\langle L_{r_{0} M_{S_{0}}} M_{L_{0}} S_{0} M_{S_{0}} \mid J_{0} M_{0}\right\rangle \times \sum_{m m^{\prime} m_{s} m^{\prime} s}\left\langle l^{\prime} m^{\prime} l-m \mid L_{0} M_{L_{0}}\right\rangle \\
\times\left\langle\frac{1}{2} m_{s}^{\prime} \frac{1}{2}-m_{s} \mid S_{0} M_{S_{0}}\right\rangle a_{(\eta 0) l^{\prime} m^{\prime} m^{\prime} s}^{\dagger} a_{(\eta 0) l m m_{s}}(-1)^{n+l-m+\frac{1}{2}-m_{s}} .
\end{aligned}
$$

In terms of these spherical tensor operators, one-body operators can be expressed in terms of SU(3) irreducible tensor operators, $T^{\left(\lambda_{0} \mu_{0}\right) x_{0} L_{0} S_{0} J_{0}} \underset{M_{0}}{ }$, by

$$
T^{\left(\lambda_{0} \mu_{0}\right) \kappa_{0} L_{0} S_{0} J_{0}}=\sum_{M_{0}}\left\langle(\eta 0) l^{\prime} ;(0 \eta) l \|\left(\lambda_{0} \mu_{0}\right) \kappa_{0} L_{0}\right\rangle T\left(l^{\prime}, l\right)^{L_{0} S_{0} J_{0}},
$$

where the double-barred coefficient is a reduced $\mathrm{SU}(3) \supset \mathbf{R}(3)$ Wigner coefficient [refs. $\left.{ }^{14,15}\right)$ ] for the orthonormal basis $\left|(\lambda \mu) \kappa L M_{L}\right\rangle$, where the Elliott label $K$ has been replaced by the label $\kappa$ introduced by Vergados ${ }^{15}$ ) to construct an orthonormal basis which is tied as closely as possible to the Elliott states. [For $\lambda \geqq \mu, \kappa=\mu$, $\mu-2, \ldots$. For fixed $\mu$ the states $\left|(\lambda \mu) \kappa L M_{L}\right\rangle$ go over into the states $\left|(\lambda \mu) K L M_{L}\right\rangle$ in the limit $\lambda \rightarrow \infty$. Phase conventions will follow refs. ${ }^{13,14}$ ) rather than ref. ${ }^{15}$ ). Note the factor $(-1)^{\eta}$ in eq. (1).]

The decomposition into irreducible tensor components in the pseudo space will be illustrated by the magnetic moment operator

$$
\boldsymbol{\mu}=g_{l} \boldsymbol{J}+\left(g_{s}-g_{l}\right) \boldsymbol{S} .
$$

Here, $\boldsymbol{J}=\tilde{\boldsymbol{L}}+\tilde{\boldsymbol{S}}$ as well as $\boldsymbol{J}=\boldsymbol{L}+\boldsymbol{S}$, but since $\boldsymbol{S}$ has no simple form in the pseudo space it is necessary to transform the spin operator $S$ to the pseudo coupling scheme for the appropriate proton or neutron subspace of the full shell-model space. By 
means of recoupling transformations from $l-s \rightarrow j-j \rightarrow l-\tilde{s}$ coupling it can be shown that

$$
\mu=g_{l} J-\frac{1}{3}\left(g_{s}-g_{l}\right) \tilde{S}+\left(g_{s}-g_{l}\right) \sum_{\tilde{l} \tilde{l}} \mu_{i} \tilde{i} \tau\left(l^{\prime}, l\right)^{\tilde{L}_{0}=2, \tilde{s}_{0}=1, J_{0}=1},
$$

where the last term is a spherical tensor of rank 2 in pseudo orbital space and rank 1 in pseudo spin space, and the coefficients $\mu_{i \tilde{i}}$ are given by

$$
\begin{aligned}
\mu_{i \tilde{i}}=\sum_{j^{\prime} j}\left[\frac{\left(2 j^{\prime}+1\right)(2 j+1)}{2}\right]^{\frac{1}{2}}(-1)^{l-j+\frac{1}{2}}\left\{\begin{array}{lll}
j & \frac{1}{2} & l \\
\frac{1}{2} & j^{\prime} & 1
\end{array}\right\}\left[\left(2 j^{\prime}+1\right)(2 j+1) \times 3 \times 5\right]^{\frac{1}{2}} & \\
& \times(-1)^{\tilde{\eta}}\left\{\begin{array}{ccc}
l & \frac{1}{2} & j \\
\tilde{L}_{0} & S_{0}^{\frac{1}{2}} & j^{\prime} \\
L_{0}=2 & 1
\end{array}\right\} .
\end{aligned}
$$

Finally, the complicated tensor term can be transformed to SU(3) irreducible tensor form, (by the inverse of eq. (3)), to yield

$$
\boldsymbol{\mu}=g_{l} J-\frac{1}{3}\left(g_{s}-g_{l}\right) \tilde{S}+\left(g_{s}-g_{l}\right) \sum_{\left(\tilde{\lambda}_{0} \tilde{\mu}_{0}\right) \tilde{x}_{0}} \mu_{\left(\tilde{\lambda}_{0} \tilde{\mu}_{0}\right) \tilde{x}_{0}} T^{\left(\tilde{\lambda}_{0} \tilde{\mu}_{0}\right) \tilde{x}_{0} \tilde{L}_{0}=2, \tilde{s}_{0}=1, J_{0}=1},
$$

\begin{tabular}{|c|c|c|c|c|c|}
\hline \multicolumn{6}{|c|}{$\bar{N}=3$ shell } \\
\hline$l^{\prime}$ & $l$ & $\mu_{\tilde{i} \tilde{i} \boldsymbol{i}}$ & $\left(\tilde{\lambda}_{0} \tilde{\mu}_{0}\right)$ & $\tilde{\kappa}_{0}$ & $\mu_{\left(\tilde{\lambda}_{0} \tilde{\mu}_{0}\right) \tilde{x}_{0}}$ \\
\hline \multirow[t]{2}{*}{1} & 1 & $-\left(\frac{8}{15}\right)^{\frac{1}{2}}$ & (11) & 1 & 1.71402 \\
\hline & & & (22) & 0 & -0.04926 \\
\hline 1 & 3 & $\left(\frac{4}{5}\right)^{\frac{1}{2}}$ & (22) & 2 & 0.10096 \\
\hline 3 & 1 & & (33) & 1 & 0.11164 \\
\hline 3 & 3 & $-\frac{4}{3}\left(\frac{7}{15}\right)^{\frac{1}{2}}$ & & & \\
\hline \multicolumn{6}{|c|}{$\tilde{N}=4$ shell } \\
\hline$l^{\prime}$ & $l$ & $\mu_{\tilde{i} \tilde{l}}$ & $\left(\tilde{\lambda}_{\circ} \tilde{\mu}_{0}\right)$ & $\tilde{\kappa}_{0}$ & $\mu_{\left(\tilde{\lambda}_{0} \tilde{\mu}_{0}\right) \tilde{\kappa}_{0}}$ \\
\hline \multirow[t]{2}{*}{2} & 2 & $\frac{2}{3}\left(\frac{10}{7}\right)^{\frac{1}{2}}$ & (11) & 1 & -2.18562 \\
\hline & & & (22) & 0 & 0.07147 \\
\hline 2 & 4 & $-\left(\frac{8}{7}\right)^{\frac{1}{2}}$ & (22) & 2 & -0.14648 \\
\hline \multirow[t]{2}{*}{4} & 2 & & (33) & 1 & -0.21196 \\
\hline & & & (44) & 0 & 0.00382 \\
\hline 4 & 4 & $\left(\frac{80}{77}\right)^{\frac{1}{2}}$ & (44) & 2 & 0.13470 \\
\hline 0 & 2 & & & & \\
\hline 2 & 0 & $-\frac{2}{3}$ & & & \\
\hline
\end{tabular}

with $\left(\tilde{\lambda}_{0} \tilde{\mu}_{0}\right)=(11),(22), \ldots,(\tilde{\eta} \tilde{\eta})$. The coefficients $\mu_{\tilde{l} \tilde{l}^{\prime}}$ and $\mu_{\left(\tilde{\lambda}_{0} \tilde{\mu}_{0}\right) \tilde{\kappa}_{0}}$ are given in

TABLE 6

Coefficients for the tensor term of $\mu$ 
table 6 for the shells $\tilde{N}(=\tilde{\eta})=3$ and $\tilde{N}(=\tilde{\eta})=4$, needed for the odd- $Z$ and odd- $N$ nuclei of the rare-earth region.

The one-body $\boldsymbol{l} \cdot \boldsymbol{s}$ and $\boldsymbol{l}^{2}$ operators (or $\tilde{l} \cdot \tilde{s}$ and $\tilde{l}^{2}$ ) can also be transformed to $\mathrm{SU}(3)$ irreducible tensor form.

$$
\begin{gathered}
\sum_{i=1}^{n} l_{i} \cdot s_{i}=-\sum_{l\left(\lambda_{0} \mu_{0}\right)}(-1)^{\eta}\left[\frac{1}{2} l(l+1)(2 l+1)\right]^{\frac{1}{2}}\left\langle(\eta 0) l ;(0 \eta) l \|\left(\lambda_{0} \mu_{0}\right) \kappa_{0} L_{0}=1\right\rangle \\
\times T^{\left(\lambda_{0} \mu_{0}\right) \kappa_{0} L_{0}=1, s_{0}=1, J_{0}=0,}, \\
\begin{aligned}
\sum_{i=1}^{n} l_{i}^{2}=\sum_{l\left(\lambda_{0} \mu_{0}\right)}(-1)^{\eta} l(l+1)[2(2 l+1)]^{\frac{1}{2}}\left\langle(\eta 0) l ;(0 \eta) l \|\left(\lambda_{0} \mu_{0}\right) \kappa_{0}=L_{0}=0\right\rangle \\
\times T^{\left(\lambda_{0} \mu_{0}\right) \kappa_{0}=L_{0}=S_{0}=J_{0}=0} .
\end{aligned}
\end{gathered}
$$

For the $\eta=3$ shell, in particular, these have the form

$$
\begin{gathered}
\sum_{i=1}^{n} l_{i} \cdot s_{i}=-3 \sqrt{5} T^{(11) x_{0}=1, L_{0}=1, S_{0}=1, J_{0}=0}, \\
\sum_{i=1}^{n} l_{i}^{2}=18 \sqrt{5} T^{(00) \kappa_{0}=L_{0}=S_{0}=J_{0}=0}+2 \sqrt{105} T^{(22) \kappa_{0}=L_{0}=S_{0}=J_{0}=0}
\end{gathered}
$$

Two-body operators can be expressed in terms of SU(3) irreducible tensor operators

$$
T^{\left[\left(\lambda^{\prime} \mu_{2} \mu_{2}\right)\left(\mu_{2} \lambda_{2}\right)\right]\left(\lambda_{0} \mu_{0}\right) \rho_{0} \kappa_{0} L_{0}} \mathcal{L}_{\mathcal{L}_{0} M^{\prime} s_{0}},
$$

which are obtained by coupling the irreducible tensor pair-creation operators

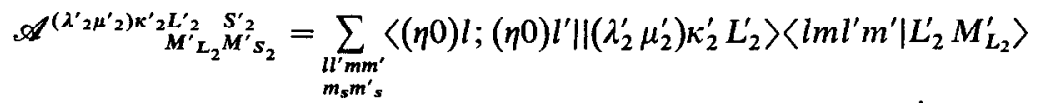

$$
\begin{aligned}
& \times\left\langle\frac{1}{2} m_{\mathrm{s}} \frac{1}{2} m_{\mathrm{s}}^{\prime} \mid S_{2}^{\prime} M_{S_{2}}^{\prime}\right\rangle a_{(\eta 0) l m m_{s}}^{\dagger} a_{(\eta 0) l^{\prime} m^{\prime} m^{\prime} s}^{\dagger},
\end{aligned}
$$

to the pair annihilation operators, (using the tensor form of eq. (1))

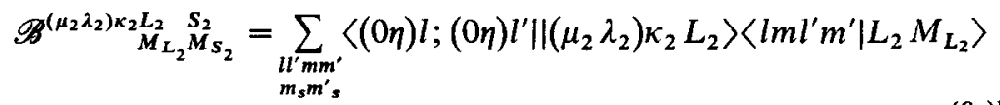

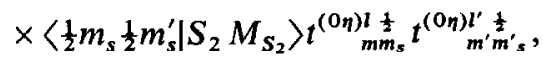

$$
\begin{aligned}
& T^{\left[\left(\lambda^{\prime} \mu^{\prime} \mu_{2}^{\prime}\right)\left(\mu_{2} \lambda_{2}\right)\right]\left(\lambda_{0} \mu_{0}\right) \rho_{0} x_{0} L_{0}} S_{M_{L_{2}} M_{S_{0}}}
\end{aligned}
$$

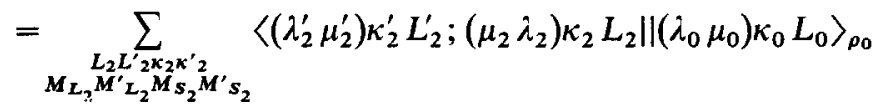

$$
\begin{aligned}
& \times\left\langle L_{2}^{\prime} M_{L_{2}}^{\prime} L_{2} M_{L_{2}} \mid L_{0} M_{L_{0}}\right\rangle\left\langle S_{2}^{\prime} M_{S_{2}}^{\prime} S_{2} M_{S_{2}} \mid S_{0} M_{S_{0}}\right\rangle
\end{aligned}
$$

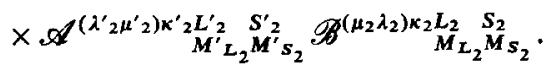

The multiplicity label $\rho_{0}$ is needed in those cases where the direct product $\left(\lambda_{2}^{\prime} \mu_{2}^{\prime}\right) \times$ $\left(\mu_{2} \lambda_{2}\right)$ contains the irreducible representation $\left(\lambda_{0} \mu_{0}\right)$ more than once, see refs. $\left.{ }^{13-15}\right)$. In the $\eta=3$ shell the two-particle representations are $\left(\lambda_{2} \mu_{2}\right)=(60),(41),(22),(03)$. Here, (60) and (22) correspond to a symmetric coupling of the two single-particle representations (30) and hence two-particle spin $S_{2}=0$, (or $\tilde{S}_{2}=0$ ), while (41) and (03), correspond to an antisymmetric coupling of the two single-particle representa- 
TABLE 7

Irreducible SU(3) tensor character of the surface delta interaction in the $\tilde{N}=3$ shell

\begin{tabular}{cccc}
\hline$\left(\lambda_{2}^{\prime} \mu_{2}^{\prime}\right)$ & $\left(\mu_{2} \lambda_{2}\right)$ & $\left(\lambda_{0} \mu_{0}\right) \rho_{0}$ & $\left.\left.W_{\left(\left[\lambda^{\prime}{ }_{2} \mu^{\prime}{ }_{2}\right)\right.}\left(\mu_{2} \lambda_{2}\right)\right]\left(\lambda_{0} \mu_{0}\right) \rho_{0} \alpha_{0}=0 L_{0}=0\right)$ \\
\hline$(60)$ & $(06)$ & $(00)$ & 8.46417 \\
$(22)$ & $(22)$ & $(00)$ & 1.00298 \\
$(60)$ & $(06)$ & $(22)$ & -4.36981 \\
$(60)$ & $(22)$ & $(22)$ & 3.64717 \\
$(22)$ & $(06)$ & $(22)$ & 3.64717 \\
$(22)$ & $(22)$ & $(22) 1$ & -0.76016 \\
$(22)$ & $(22)$ & $(22) 2$ & 0.37014 \\
$(22)$ & $(22)$ & $(22) 3$ & -1.26958 \\
$(60)$ & $(06)$ & $(44)$ & 0.59215 \\
$(60)$ & $(22)$ & $(44)$ & -1.00955 \\
$(22)$ & $(06)$ & $(44)$ & -1.00955 \\
$(22)$ & $(22)$ & $(44)$ & 0.23733 \\
$(60)$ & $(06)$ & $(66)$ & -0.16154 \\
$(60)$ & $(22)$ & $(82)$ & -0.08405 \\
$(22)$ & $(06)$ & $(28)$ & 0.08405 \\
$(60)$ & $(22)$ & $(60)$ & 0.54752 \\
$(22)$ & $(06)$ & $(06)$ & -0.54752 \\
$(22)$ & $(22)$ & $(60)$ & -0.42778 \\
$(22)$ & $(22)$ & $(06)$ & 0.42778 \\
\hline
\end{tabular}

tions (30) and hence two-particle spin $S_{2}=1$, (or $\widetilde{S}_{2}=1$ ). Since the surface delta interaction (SDI) ${ }^{16,17}$ ) has proved to be a remarkably good effective interaction in many regions of the periodic table, particularly for highly truncated subspaces of the full shell-model space, it is natural to make a first trial energy calculation with this very simple two-body interaction. In configurations of identical particles the SDI acts only on two-particle states coupled to $S=0$, in the case of both the real or the pseudo coupling schemes ${ }^{17}$ ). In the $\eta=3$ shell therefore only two-body tensor terms with $\left(\lambda_{2} \mu_{2}\right)=(60)$ and (22) survive for the SDI. The most general two-body interaction is expanded in the form

$$
\begin{aligned}
H_{2-\text { body }}=\sum_{\substack{\left(\lambda^{\prime} \mu^{\prime}{ }^{\prime}\right)\left(\mu_{2} \lambda_{2} \lambda_{2}\right) \\
\left(\lambda_{0} \mu_{0}\right) \kappa_{0} \rho_{0} L_{0}}} W\left(\left[\left(\lambda_{2}^{\prime} \mu_{2}^{\prime}\right)\left(\mu_{2} \lambda_{2}\right)\right]\left(\lambda_{0} \mu_{0}\right) \rho_{0} \kappa_{0} L_{0}\right) \\
\times T^{\left[\left(\lambda^{\prime}{ }_{2} \mu^{\prime}{ }^{\prime}\right)\left(\mu_{2} \lambda_{2}\right)\right]\left(\lambda_{0} \mu_{0}\right) \rho_{0} \kappa_{0} L_{0}=s_{0}, J_{0}=0 .}
\end{aligned}
$$

For a central interaction $L_{0}=S_{0}=0$, and hence also $\kappa_{0}=0$. The coefficients $W$ for the SDI in the $\eta=3$ shell are given in table 7 . (The coefficients are for unit strength factor, $G=1$; see, e.g. eq. (8) of ref. ${ }^{17}$ ).)

Many-particle states for the $n$-particle configurations and the $n-1$ and $n-2$ particle parent states in the fractional parentage expansions will be expressed in the $U(s) \supset$ $\mathrm{SU}(3) \supset \mathrm{R}(3)$ scheme where $s=\frac{1}{2}(\tilde{N}+1)(\tilde{N}+2)$ is the degeneracy number of the major pseudo oscillator shell. Irreducible representations of $\mathrm{U}(s)$ are specified by the partition numbers $[f]=\left[f_{1} f_{2} \ldots f_{s}\right]$ with $f_{i} \leqq 2$ (identical particles), where, for each $n,[f]$ can also be characterized by the total pseudo spin $\tilde{S},[f]=\left[2^{\frac{1 n}{n}-\tilde{s}} 1^{2 \tilde{s}}\right]$. 
For $n \leqq 6$, the branching rules $\mathrm{U}(10) \rightarrow \mathrm{SU}(3)$ can be read from the tables of plethysms ${ }^{18}$ ) published by Ibrahim ${ }^{19}$ ), [see also ref. ${ }^{20}$ )]. We are interested mainly in pseudo $S U(3)$ representations near the leading representations. To determine the number of occurrences of a given $(\tilde{\mu} \tilde{\mu})$ near the leading $(\tilde{\lambda} \tilde{\mu})$ a simple counting process is usually sufficient. To calculate matrix elements for the leading representation $(\tilde{\lambda} \tilde{\mu})=(11,2)$ for $n=7, \tilde{S}=\frac{1}{2}\left([f]=\left[2^{3} 1\right]\right)$, for example, only the irreducible representations $(\tilde{\lambda} \tilde{\mu})$ with $2 \tilde{\lambda}+\tilde{\mu} \geqq 18$ for $n=6$ and $2 \tilde{\lambda}+\tilde{\mu} \geqq 12$ for $n=5$ are needed. To within these limits the needed branching rules $\mathrm{U}(10) \rightarrow \mathrm{SU}(3)$ for $n=6$ and $n=5$ are given by

$$
\begin{aligned}
& {\left[2^{3}\right](\tilde{S}=0) \rightarrow(\underline{12,0})(\underline{93})(66)(74)(82)^{3} \ldots,} \\
& {\left[2^{2} 1^{2}\right](\tilde{S}=1) \rightarrow(\underline{93})(\underline{10,1})(74)^{2}(\underline{82})^{2}(90) \ldots,} \\
& {\left[2^{2} 1\right]\left(\tilde{S}=\frac{1}{2}\right) \rightarrow(\underline{10,1})(\underline{74})(\underline{82})(\underline{90})(55)(\underline{63})^{3}(71)^{2}(36)(44)^{3}(\underline{52})^{4}(60) \ldots,} \\
& {\left[21^{3}\right]\left(\tilde{S}=\frac{3}{2}\right) \rightarrow(\underline{82})(\underline{63})^{2}(\underline{71})(36)(44)^{2}(\underline{52})^{2}(60)^{2} \ldots}
\end{aligned}
$$

Only the states underlined have a fractional parentage connection to the $(11,2)$ state of $\left[2^{3} 1\right]$.

State vectors are specified by $\mid[f] \alpha(\lambda \mu) \kappa L S J M>$ where $\alpha$ is a label used to distinguish the different states $(\lambda \mu)$ contained in a given $[f]$ in those cases involving multiple occurrences. (Superscripts ( ${ }^{\sim}$ ) will again be omitted for brevity.) Fractional parentage coefficients are defined in terms of reduced matrix elements of the singleparticle creation operators

$$
\begin{aligned}
& \left\langle\left[f^{\prime}\right] \alpha^{\prime}\left(\lambda^{\prime} \mu^{\prime}\right) \kappa^{\prime} L^{\prime} M_{L}^{\prime} S^{\prime} M_{S}^{\prime}\left|a_{(\eta 0) \lim \frac{1}{s}}^{\dagger}\right|[f] \alpha(\lambda \mu) \kappa L M_{L} S M_{S}\right\rangle \\
& =\left\langle\left[f^{\prime}\right] \alpha^{\prime}\left(\lambda^{\prime} \mu^{\prime}\right) S^{\prime}\left\|\mid a_{(\eta 0) \frac{1}{2}}^{\dagger}\right\|[[f] \alpha(\lambda \mu) S\rangle\left\langle(\lambda \mu) \kappa L ;(\eta 0) l \|\left(\lambda^{\prime} \mu^{\prime}\right) \kappa^{\prime} L^{\prime}\right\rangle\right. \\
& \quad \times\left\langle L M_{L} l m \mid L M_{L}^{\prime}\right\rangle\left\langle S M_{S} \frac{1}{2} m_{s} \mid S^{\prime} M_{S}^{\prime}\right\rangle
\end{aligned}
$$

where the notation of a triple-barred matrix element has been introduced, first to indicate that both the dependence on $M$ quantum numbers and SU(3) subgroup labels $\kappa$ and $L$ have been removed, the latter by means of the reduced $\mathrm{SU}(3) \supset \mathrm{R}(3)$ Wigner coefficient; and second to distinguish it from double-barred reduced matrix elements used by some authors which include in their definition additional square roots of dimension factors. Except for a factor of $\sqrt{ } n$, these triple-barred reduced matrix elements are $n \rightarrow n-1$ coefficients of fractional parentage (c.f.p.), more specifically the $\mathrm{U}(10) \supset \mathrm{SU}(3)$ factors of the full c.f.p.

These c.f.p. have been calculated for the leading SU(3) representations for several values of $n$ by a slight generalization of the technique suggested in ref. ${ }^{21}$ ). Full details and tabulations are given in ref. ${ }^{12}$ ). The triple-barred reduced matrix elements satisfy the sum rule

$$
\sum_{\alpha(\lambda \mu)}\left\langle[ f ^ { \prime } ] \alpha ^ { \prime } ( \lambda ^ { \prime } \mu ^ { \prime } ) S ^ { \prime } \left\|\left|a_{(\eta 0)+\frac{1}{2}}^{\dagger} \|[f] \alpha(\lambda \mu) S\right\rangle^{2}=n \frac{\operatorname{dim}[f]}{\operatorname{dim}\left[f^{\prime}\right]},\right.\right.
$$

where $\operatorname{dim}[f]$ and $\operatorname{dim}\left[f^{\prime}\right]$ are the dimensions of the irreducible representations $[f]$ 
and $\left[f^{\prime}\right]$ of the permutation groups for $n-1$ and $n$ particles, respectively. If the summation in eq. (15) includes besides $(\lambda \mu)$ and $\alpha$ a sum over all possible [ $f$ ] (hence $S$ ) the value of the sum is simply given by $n$, the number of particles.

In terms of these c.f.p. the matrix element of a one-body irreducible tensor operator of the type introduced in eq. (3) is given by the c.f.p. expansion

$$
\begin{aligned}
& \left\langle\left[f^{\prime \prime}\right] \alpha^{\prime \prime}\left(\lambda^{\prime \prime} \mu^{\prime \prime}\right) \kappa^{\prime \prime} L^{\prime \prime} S^{\prime \prime} J^{\prime \prime} M^{\prime \prime}\left|T^{\left(\lambda_{0} \mu_{0}\right) \kappa_{0} L_{0} S_{0} J_{0}}\right|[f] \alpha(\lambda \mu) \kappa L S J M\right\rangle \\
& =\left\langle J M J_{0} M_{0} \mid J^{\prime \prime} M^{\prime \prime}\right\rangle\left[(2 J+1)\left(2 J_{0}+1\right)\left(2 L^{\prime \prime}+1\right)\left(2 S^{\prime \prime}+1\right)\right]^{\frac{1}{2}}\left\{\begin{array}{ccc}
L & L_{0} & L^{\prime \prime} \\
S & S_{0} & S^{\prime \prime} \\
J & J_{0} & J^{\prime \prime}
\end{array}\right\} \\
& \times \sum_{\left[\delta^{\prime}\right] S^{\prime} \alpha^{\prime}\left(\lambda^{\prime} \mu^{\prime}\right)}(-1)^{S^{\prime}+S^{\prime \prime}+\frac{1}{2}-S_{0}}\left[(2 S+1)\left(2 S_{0}^{\prime}+1\right)\right]^{\frac{1}{2}}\left\{\begin{array}{ccc}
S & \frac{1}{2} & S^{\prime} \\
\frac{1}{2} & S^{\prime \prime} & S_{0}
\end{array}\right]\left[\frac{\mathrm{d}(\lambda \mu)}{\mathrm{d}\left(\lambda^{\prime} \mu^{\prime}\right)}\right]^{\frac{1}{2}} \\
& \times(-1)^{\eta+\lambda_{0}-\mu_{0}+\lambda^{\prime}-\mu^{\prime}-\lambda+\mu}\left\langle\left[f^{\prime \prime}\right] \alpha^{\prime \prime}\left(\lambda^{\prime \prime} \mu^{\prime \prime}\right) S^{\prime \prime}||\right| a_{(\eta 0) \frac{1}{2}}^{+} \|\left[\left[f^{\prime}\right] \alpha^{\prime}\left(\lambda^{\prime} \mu^{\prime}\right) S^{\prime}\right\rangle \\
& \times\left\langle[f] \alpha(\lambda \mu) S\|\| a_{(\eta 0) \frac{1}{2}}^{\dagger} \|\left[\left[f^{\prime}\right] \alpha^{\prime}\left(\lambda^{\prime} \mu^{\prime}\right) S^{\prime}\right\rangle\right. \\
& \times \sum_{\rho}\left\langle(\lambda \mu) \kappa L ;\left(\lambda_{0} \mu_{0}\right) \kappa_{0} L_{0} \|\left(\lambda^{\prime \prime} \mu^{\prime \prime}\right) \kappa^{\prime \prime} L^{\prime \prime}\right\rangle_{\rho} U\left((\lambda \mu)(0 \eta)\left(\lambda^{\prime \prime} \mu^{\prime \prime}\right)(\eta 0) ;\left(\lambda^{\prime} \mu^{\prime}\right)\right. \text {; } \\
& \left.\left(\lambda_{0} \mu_{0}\right) \rho\right) \text {, }
\end{aligned}
$$

where a generalized Wigner-Eckart theorem was been used. Besides the summation over $M$ quantum numbers, which leads to the usual angular momentum recoupling coefficients, the summation over $\mathrm{SU}(3)$ subgroup labels $\kappa$ and $L$ has been performed as well, so that the final result is weighted with analogous $\mathrm{SU}(3)$ Racah coefficients. Here $\left.U(\lambda \mu)(0 \eta)\left(\lambda^{\prime \prime} \mu^{\prime \prime}\right)(\eta 0) ;\left(\lambda^{\prime} \mu^{\prime}\right) ;\left(\lambda_{0} \mu_{0}\right) \rho\right)$ is an $\mathrm{SU}(3)$ Racah coefficient in unitary form, as defined in ref. $\left.{ }^{21}\right)$; and $\mathrm{d}(\lambda \mu)$ stands for the dimension of the irreducible representation $(\lambda \mu)$. The final product of SU(3) Wigner and Racah coefficients involves a sum over the multiplicity label $\rho$ for the coupling $(\lambda \mu) \times\left(\lambda_{0} \mu_{0}\right) \rightarrow\left(\lambda^{\prime \prime} \mu^{\prime \prime}\right)$.

Matrix elements of two-body operators, eqs. (12) and (13), are given by similar c.f.p. expansions. For the case of a central interaction, for example, with $L_{0}=S_{0}=0$, the matrix element of the two-body irreducible tensor component is given by

$$
\begin{aligned}
& \left\langle\left[f^{\prime \prime}\right] \alpha^{\prime \prime}\left(\lambda^{\prime \prime} \mu^{\prime \prime}\right) \kappa^{\prime \prime} L S J M\left|T^{\left[\left(\lambda^{\prime} \mu^{\prime} 2\right)\left(\mu_{2} \lambda_{2}\right)\right]\left(\lambda_{0} \mu_{0}\right) \rho_{0} \alpha_{0} 000}\right|[f] \alpha(\lambda \mu) \kappa L S J M\right\rangle \\
& =-n(n-1) \sum_{\left[f_{n-2}\right] \alpha_{n-2}\left(\lambda_{n-2} \mu_{n-2}\right)} \sum_{\rho_{2} \rho_{2}^{\prime} 2}\left[\frac{\mathrm{d}(\lambda \mu)}{\mathrm{d}\left(\lambda_{n-2} \mu_{n-2}\right)}\right]^{\frac{1}{2}} \\
& \quad \times(-1)^{\lambda^{\prime} 2+\mu_{2}^{\prime}-\lambda-\mu-\lambda_{0}-\mu_{0}+\lambda_{n-2}+\mu_{n-2}+\rho_{0}-\rho_{0} \max } \\
& \left.\left.\quad \times\left\langle\left[f_{n-2}\right] \alpha_{n-2}\left(\lambda_{n-2} \mu_{n-2}\right) S_{n-2} ;\left[f_{2}^{\prime}\right]\left(\lambda_{2}^{\prime} \mu_{2}^{\prime}\right) S_{2}^{\prime} \rho_{2}^{\prime}\right|\right\}\left[f^{\prime \prime}\right] \alpha^{\prime \prime}\left(\lambda^{\prime \prime} \mu^{\prime \prime}\right) S^{\prime \prime}\right\rangle \\
& \left.\left.\quad \times\left\langle\left[f_{n-2}\right] \alpha_{n-2}\left(\lambda_{n-2} \mu_{n-2}\right) S_{n-2} ;\left[f_{2}\right]\left(\lambda_{2} \mu_{2}\right) S_{2} \rho_{2}\right|\right\}[f] \alpha(\lambda \mu) S\right\rangle \\
& \quad \times \sum_{\rho}\left\langle(\lambda \mu) \kappa L ;\left(\lambda_{0} \mu_{0}\right) \kappa_{0}=L_{0}=0||\left(\lambda^{\prime \prime} \mu^{\prime \prime}\right) \kappa^{\prime \prime} L\right\rangle_{\rho} U\left(\left(\lambda^{\prime \prime} \mu^{\prime \prime}\right)\left(\mu_{2}^{\prime} \lambda_{2}^{\prime}\right)(\lambda \mu)\left(\lambda_{2} \mu_{2}\right) ;\right. \\
& \left.\quad\left(\lambda_{n-2} \mu_{n-2}\right) \rho_{2}^{\prime} \rho_{2} ;\left(\mu_{0} \lambda_{0}\right) \rho_{0} \rho\right) .
\end{aligned}
$$

The $n \rightarrow n-2$ c.f.p. can be related to triple-barred reduced matrix elements of the 
pair-creation operators of eq. (10)

$$
\begin{aligned}
& \left.\left.\left\langle\left[f_{n-2}\right] \alpha_{n-2}\left(\lambda_{n-2} \mu_{n-2}\right) S_{n-2} ;\left[f_{2}\right]\left(\lambda_{2} \mu_{2}\right) S_{2} \rho_{2}\right|\right\}[f] \alpha(\lambda \mu) S\right\rangle \\
& \quad=\left[\frac{2}{n(n-1)}\right]^{\frac{1}{2}}\left\langle[f] \alpha(\lambda \mu) S||\left|\mathscr{A}\left(\lambda_{2} \mu_{2}\right) S_{2} \rho_{2}\right|||\left[f_{n-2}\right] \alpha_{n-2}\left(\lambda_{n-2} \mu_{n-2}\right) S_{n-2}\right\rangle,
\end{aligned}
$$

where the multiplicity label $\rho_{2}$ refers to the coupling $\left(\lambda_{n-2} \mu_{n-2}\right) \times\left(\lambda_{2} \mu_{2}\right) \rightarrow(\lambda \mu)$. These $n \rightarrow n-2$ c.f.p. can be calculated directly from the triple-barred reduced matrix elements by a generalization of the technique outlined in ref. ${ }^{21}$ ) or can be compounded ${ }^{12}$ ) from the $n \rightarrow n-1$ particle reduced matrix elements by the relation

$$
\begin{aligned}
& \left.\left.\left\langle\left[f_{n-2}\right] \alpha_{n-2}\left(\lambda_{n-2} \mu_{n-2}\right) S_{n-2} ;\left[f_{2}\right]\left(\lambda_{2} \mu_{2}\right) S_{2} \rho_{2}\right|\right\}[f] \alpha(\lambda \mu) S\right\rangle \\
& =\frac{1}{[n(n-1)]^{\frac{1}{2}}} \sum_{S_{n-1}\left(\left[f_{n-1}\right]\right)} U\left(S_{n-2} \frac{1}{2} S_{\frac{1}{2}}^{1} ; S_{n-1} S_{2}\right) \\
& \quad \times \sum_{\left(\lambda_{n-1} \mu_{n-1}\right) \alpha_{n-1}}\left\langle[f] \alpha(\lambda \mu) S||\left|a_{(\eta 0) \frac{1}{2}}^{\dagger} \|\right| \mid\left[f_{n-1}\right] \alpha_{n-1}\left(\lambda_{n-1} \mu_{n-1}\right) S_{n-1}\right\rangle \\
& \quad \times\left\langle\left[f_{n-1}\right] \alpha_{n-1}\left(\lambda_{n-1} \mu_{n-1}\right) S_{n-1}||\left|a_{(\eta 0) \frac{1}{2}}^{\dagger}\right| \mid\left[f_{n-2}\right] \alpha_{n-2}\left(\lambda_{n-2} \mu_{n-2}\right) S_{n-2}\right\rangle \\
& \quad \times U\left(\left(\lambda_{n-2} \mu_{n-2}\right)(n 0)(\lambda \mu)(\eta 0) ;\left(\lambda_{n-1} \mu_{n-1}\right) ;\left(\lambda_{2} \mu_{2}\right) \rho_{2}\right) .
\end{aligned}
$$

\section{Magnetic moments}

Magnetic moments of the positive-parity states of odd- $Z$ nuclei and negative-parity states of odd- $N$ nuclei in the rare-earth region may be understood in terms of the leading pseudo $\mathrm{SU}(3)$ representations of the configurations $\left(\mathrm{g}_{\frac{7}{2}} \mathrm{~d}_{\frac{3}{2}} \mathrm{~d}_{\frac{3}{2}} \mathrm{~s}_{\frac{1}{3}}\right)^{n}=(\tilde{\mathrm{f}} \tilde{\mathrm{p}})^{n}$ and $\left(h_{\frac{3}{2}} f_{\frac{3}{3}} f_{\frac{3}{2}} p_{\frac{3}{2}} p_{\frac{1}{3}}\right)^{n}=(\tilde{g} \tilde{d})^{n}$, respectively. Similar descriptions may be valid for the magnetic moments of the natural-parity states in the actinides.

The magnetic moment operator has been transformed to the pseudo coupling scheme in sect. 2 and is given in appropriate form by eq. (7)

$$
\boldsymbol{\mu}=g_{l} \boldsymbol{J}-\frac{1}{3}\left(g_{s}-g_{l}\right) \tilde{\boldsymbol{S}}+\left(g_{s}-g_{l}\right) \sum_{\left(\lambda_{0} \tilde{\mu}_{0}\right) \tilde{\kappa}_{0}} \mu_{\left(\tilde{\tilde{\alpha}}_{0} \tilde{\mu}_{0}\right) \tilde{\kappa}_{0}} T^{\left(\tilde{\lambda}_{0} \tilde{\mu}_{0}\right) \tilde{\kappa}_{0} \tilde{L}_{0}=2, \tilde{s}_{0}=1, J_{0}=1} .
$$

The first two terms depend only on the total angular momentum operator $J$ and the total pseudo spin operator $\tilde{\boldsymbol{S}}$, and since $\boldsymbol{J}=\tilde{\boldsymbol{L}}+\widetilde{\boldsymbol{S}}$ their matrix elements are simple functions of $\tilde{L}, \tilde{S}$, and $J$ only. The last term is expressed in terms of SU(3) irreducible tensor operators. It has spherical tensor rank 2 in pseudo orbital space and rank 1 in pseudo spin space. Its $\mathrm{SU}(3)$ irreducible tensor character is dominated by the component with $\left(\tilde{\lambda}_{0} \tilde{\mu}_{0}\right)=(11)$; but it also contains components with $\left(\tilde{\lambda}_{0} \tilde{\mu}_{0}\right)=(22), \ldots$, $(\tilde{N} \bar{N})$; (see table 6 ). The expectation values of this complicated tensor term have been calculated for the leading pseudo $S U(3)$ representations for the odd- $Z$ configurations $(\tilde{N}=3)$ for several values of $n$, using the general form of the matrix element of the SU(3) irreducible tensor operators given by eq. (16). Results are shown in table 8. Although the matrix elements of this complicated tensor term might be expected to be 
small since they are weighted by both angular momentum recoupling and SU(3) Racah coefficients, they turn out to be almost completely negligible. This came as somewhat of a surprise. Some of it can be understood in terms of the large values of the quantum number $\bar{\lambda}$ in the leading representations, since the SU(3) $\supset \mathbf{R}(3)$ coefficients in eq. (16) tend to zero for certain values of the multiplicity label $\rho$ as $\bar{\lambda} \rightarrow \infty$. However, these coefficients remain finite for at least one value of $\rho$ for each $\left(\tilde{\lambda}_{0} \tilde{\mu}_{0}\right)$. The extreme smallness of these expectation values of the complicated tensor terms is due largely to a cancellation of the many terms in the c.f.p. expansion of the matrix elements for the many-particle states. (In particular, terms come with opposite signs for parent states with $\tilde{S}_{n-1}=0$ and $\tilde{S}_{n-1}=1$ in eq. (16).) The extreme smallness of these expectation values is thus largely a many-particle effect. The single-particle state is the only state for which this expectation value is large. For $n=1,(\tilde{\lambda} \tilde{\mu})=(30)$, $\tilde{L}=1, J=\frac{1}{2}$, the expectation value of the complicated tensor term is given by $\frac{4}{9}\left(g_{s}-g_{l}\right)$, which together with the first two terms leads to the Schmidt value for the magnetic moment. Of course, for $n=1$ we would not expect to get good results in terms of this model. The systematics of the rare-earth region would lead us to expect that the pseudo SU(3) model should be good only for $n \geqq 7$. Nevertheless, the results for $n=3$ and 5 have been included in table 8 since they give an indication of the magnitude of the complicated tensor term, (and in a complete configuration-mixed description terms with $n=5$ and 3 may in fact make up very small pieces of the full state vector for $n=7$ ).

The results of table 8 suggest that the expectation value of the complicated tensor term can be neglected to good approximation, (its largest contribution has been found

TABLE 8

Contributions of tensor term to $\mu$

\begin{tabular}{clcccc}
\hline $\begin{array}{c}\text { Number of } \\
\text { natural-parity } \\
\text { protons }(n)\end{array}$ & $\begin{array}{c}\text { Leading } \\
(\tilde{\lambda} \tilde{\mu})\end{array}$ & $\tilde{K}_{L}$ & $\tilde{L}$ & $J$ & $\begin{array}{c}\text { Expectation } \\
\text { value of } \\
\text { tensor term }\end{array}$ \\
\hline 3 & $(71)$ & 1 & 1 & $\frac{3}{2}$ & $-0.005\left(g_{s}-g_{l}\right)$ \\
5 & $(10,1)$ & 1 & 1 & $\frac{1}{2}$ & $+0.039\left(g_{s}-g_{l}\right)$ \\
7 & $(11,2)$ & 2 & 2 & $\frac{5}{2}$ & $+0.004\left(g_{s}-g_{l}\right)$ \\
11 & $(11,2)$ & 0 & 1 & $\frac{1}{2}$ & $-0.038\left(g_{s}-g_{l}\right)$ \\
13 & $(93)$ & 3 & 3 & $\frac{7}{2}$ & $+0.016\left(g_{s}-g_{l}\right)$ \\
\hline
\end{tabular}

to be $0.18 \mathrm{n} . \mathrm{m}$.). To within such an approximation (that is $\pm 0.2 \mathrm{n} . \mathrm{m}$.) the magnetic moments of the natural-parity states of odd deformed nuclei might therefore be expected to be given by the simple expression

$$
\mu=g_{l} J-\frac{1}{3}\left(g_{s}-g_{l}\right) \frac{J(J+1)+\frac{3}{4}-\tilde{L}(\tilde{L}+1)}{2(J+1)},
$$

where, for the ground states, $\tilde{L}$ is determined in terms of $\widetilde{K}_{L}=\tilde{\mu}, \tilde{\mu}-2, \ldots$ by the prescription: $\tilde{L}=\widetilde{K}_{L}$ for $\widetilde{K}_{L} \neq 0$, while for $\widetilde{K}_{L}=0: \tilde{L}=0$ or 1 for $\tilde{\lambda}_{\text {even }}$ or odd. When 
more than one $\widetilde{K}_{L}$ value is possible, it is assumed that the $\widetilde{K}_{L}$ value of the ground state rotational band is given by the pseudo oscillator quantum number $\tilde{\Lambda}$ of the last occupied Nilsson orbit. In addition $\tilde{S}=\frac{1}{2}$, and the total angular momentum is given by $J=\tilde{L} \pm \frac{1}{2}$. Tables 9 and 10 compare the predictions of eq. $(20)$ with the experimentally observed values of the magnetic moments of the natural-parity states of deformed nuclei. Despite the extreme simplicity of the model, the predictions are in remarkably good agreement with the experimental values, and in many cases are in better agreement with the experimental values than the predictions of the rotational model of Bohr and Mottelson, also shown in tables 9 and 10. [Numerical values for the rotational model are taken from ref. ${ }^{22}$ ), where these have been calculated with Nilsson wave functions for deformations of appropriate magnitude, and with $g_{R}=Z / A$. It should be emphasized that these numbers for the rotational model are also for the bare zeroth-order predictions of a simple model. Improvements pos-

TABLE 9

Magnetic moments of odd-proton nuclei

\begin{tabular}{|c|c|c|c|c|}
\hline \multirow[t]{2}{*}{ Nucleus } & \multirow{2}{*}{$\begin{array}{l}\text { Ground state } \\
\text { spin }\end{array}$} & \multicolumn{3}{|c|}{$\mu$} \\
\hline & & experimental ") & predicted & rotational ${ }^{b}$ ) \\
\hline${ }_{63}^{153} \mathrm{Eu}$ & $\frac{5}{2}^{+}$ & 1.53 & 1.73 & 0.9 \\
\hline${ }_{63}^{153} \mathrm{Eu}(103 \mathrm{keV})$ & $\frac{3}{2}^{+}$ & 2.0 & 1.95 & 2.2 \\
\hline${ }_{65}^{157} \mathrm{~Tb}$ & $\frac{3}{2}^{+}$ & \pm 2.0 & 1.95 & 2.2 \\
\hline${ }_{65}^{159} \mathrm{~Tb}$ & $\frac{3}{2}^{+}$ & \pm 1.99 & 1.95 & 2.2 \\
\hline${ }_{69}^{163} \mathrm{Tm}$ & $\frac{1}{2}^{+}$ & \pm 0.08 & 0.75 & -0.2 \\
\hline${ }_{69}^{169} \mathrm{Tm}$ & $\frac{1}{2}^{+}$ & -0.232 & 0.75 & -0.2 \\
\hline${ }_{69}^{171} \mathrm{Tm}$ & $\frac{1}{2}^{+}$ & \pm 0.229 & 0.75 & -0.2 \\
\hline${ }_{71}^{175} \mathrm{Lu}$ & $\frac{7}{2}^{+}$ & 2.23 & 2.73 & 1.4 \\
\hline${ }_{71}^{177} \mathrm{Lu}$ & $\frac{7}{2}+$ & 2.24 & 2.73 & 1.4 \\
\hline${ }_{73}^{181} \mathrm{Ta}$ & $\frac{7}{2}^{+}$ & 2.36 & 2.73 & 1.5 \\
\hline${ }_{73}^{181} \mathrm{Ta}(482 \mathrm{keV})$ & $\frac{5}{2}+$ & 3.23 & 3.04 & 3.7 \\
\hline${ }_{75}^{185} \operatorname{Re}$ & $\frac{5}{2}+$ & 3.172 & 3.04 & 3.7 \\
\hline${ }_{75}^{187} \mathrm{Re}$ & $\frac{5}{2}+$ & 3.204 & 3.04 & 3.7 \\
\hline${ }_{91}^{231} \mathrm{~Pa}$ & $3^{-}$ & \pm 1.98 & 1.95 & 2.0 \\
\hline${ }_{91}^{233} \mathrm{~Pa}(27 \mathrm{~d})$ & $\frac{3}{2}-$ & 3.4 & 1.95 & 2.0 \\
\hline${ }_{93}^{237} \mathrm{~Np}(60 \mathrm{keV})$ & $\frac{5}{2}-$ & 1.8 & 1.73 & 1.0 \\
\hline${ }_{95}^{241} \mathrm{Am}$ & $\frac{5}{2}-$ & 1.59 & 1.73 & 1.0 \\
\hline${ }_{95}^{243} \mathrm{Am}$ & $\frac{5}{2}$ & 1.40 & 1.73 & 1.0 \\
\hline
\end{tabular}

a) Taken from ref. ${ }^{24}$ ).

b) Taken from ref. ${ }^{22}$ ). 
TABLE 10

Magnetic moments of odd-neutron nuclei

\begin{tabular}{|c|c|c|c|c|}
\hline \multirow[t]{2}{*}{ Nucleus } & \multirow{2}{*}{$\begin{array}{c}\text { Ground state } \\
\text { spin }\end{array}$} & \multicolumn{3}{|c|}{$\boldsymbol{\mu}$} \\
\hline & & experimental ${ }^{\mathbf{a}}$ ) & predicted & rotational ${ }^{b}$ ) \\
\hline${ }^{155} \mathrm{Gd}_{91}$ & $\frac{3}{2}^{-}$ & -0.254 & -0.38 & -0.50 \\
\hline${ }^{157} \mathrm{Gd}_{93}$ & $\frac{2}{\frac{3}{2}}-$ & -0.339 & -0.38 & -0.50 \\
\hline${ }^{161}{ }^{1} y_{95}(26 \mathrm{keV})$ & $\frac{5}{2}-$ & 0.55 & 0.63 & 1.1 \\
\hline${ }^{163} \mathrm{Dy}_{97}$ & $\frac{5}{2}-$ & 0.64 & 0.63 & 1.1 \\
\hline${ }^{163} \mathrm{Er}_{95}(75 \mathrm{~min})$ & $\frac{5}{2}-$ & 1.10 & 0.63 & 1.1 \\
\hline${ }^{165} \mathrm{Er}_{97}(10 \mathrm{~h})$ & $\frac{5}{2}-$ & \pm 0.65 & 0.63 & 1.1 \\
\hline${ }^{169} \mathrm{Er}_{101}(9.4 \mathrm{~d})$ & $\frac{1}{2}-$ & 0.513 & 0.63 & 0.7 \\
\hline${ }^{171} \mathrm{Yb}_{101}$ & $\frac{1}{2}-$ & 0.4919 & 0.63 & 0.7 \\
\hline${ }^{171} \mathrm{Er}_{103}(7.5 \mathrm{~h})$ & $\frac{5}{2}-$ & \pm 0.70 & -0.45 & -0.8 \\
\hline${ }^{173} \mathrm{Yb}_{103}$ & $\frac{5}{2}-$ & -0.6776 & -0.45 & -0.8 \\
\hline${ }^{177} \mathrm{Hf}_{105}$ & $\frac{7}{2}$ & 0.61 & 0.63 & 1.4 \\
\hline${ }^{183} W_{109}$ & $\frac{1}{2}-$ & 0.117 & -0.21 & 0.8 \\
\hline${ }^{189} \mathrm{Os}_{113}$ & $\frac{3}{2}-$ & 0.6566 & 0.63 & 0.9 \\
\hline${ }^{228} \mathrm{Th}_{139}$ & $\frac{5}{2}+$ & 0.38 & 0.63 & 0.7 \\
\hline${ }^{233} \mathrm{U}_{141}$ & $\frac{5}{2}+$ & 0.54 & 0.63 & 0.7 \\
\hline${ }^{23}{ }^{9} \mathrm{Pu}_{145}$ & $\frac{1}{2}^{+}$ & 0.20 & 0.63 & -0.1 \\
\hline${ }^{241} \mathrm{Pu}_{147}$ & $\frac{5}{2}^{+}$ & -0.73 & -0.45 & -0.5 \\
\hline
\end{tabular}

a) Taken from ref. ${ }^{24}$ ).

b) Taken from ref. ${ }^{22}$ ).

sible with the use of renormalized effective $g_{s}$ values, for example, (see ref. ${ }^{23}$ )), have not been included.] The predictions of eq. (20) have their largest deviation from the experimental values for the ${ }_{69} \mathrm{Tm}$ isotopes. In this case the entry in table $9,(0.75 \mathrm{n} . \mathrm{m}$. $)$, is for $\tilde{L}=1, J=\frac{1}{2}$, since the systematics of the region would lead us to expect a $\widetilde{K}_{L}=0$ ground state band belonging to a pseudo $\mathrm{SU}(3)$ representation with odd $\lambda$, $(\tilde{\lambda} \tilde{\mu})=(11,2)$, the leading representation for eleven active protons in the $\tilde{N}=3$ shell. In this case the complicated tensor piece of the magnetic moment operator makes one of its largest contributions, $-0.18 \mathrm{n} . \mathrm{m}$., but this is too small to bring the value of 0.75 $\mathrm{n} . \mathrm{m}$. predicted by eq. (20) into agreement with the experimental value of $-0.232 \mathrm{n} . \mathrm{m}$. for ${ }_{69}^{169} \mathrm{Tm}$. On the other hand a representation $(\tilde{\lambda} \tilde{\mu})=(82)$, one of the possible pseudo $\mathrm{SU}(3)$ representations for $n=11, \tilde{S}=\frac{1}{2}$, would have $\tilde{L}=0$ for a ground state with $J=\frac{1}{2}$; and for this $\tilde{L}$ value, eq. (20) would predict $\mu=-0.26 \mathrm{n}$.m. In this case therefore a significant admixture of this lower pseudo $\mathrm{SU}(3)$ representation would be very important. With the exception of such $\tilde{K}_{L}=0,\left(K_{J}=\frac{1}{2}\right)$, bands, however, the final result may be quite insensitive to admixtures of lower pseudo SU(3) representations since matrix elements of the complicated tensor term may again make only small contributions. The approximate validity of eq. (20) may therefore be dependent more on the goodness of the quantum numbers $\widetilde{L}$ and $\tilde{S}$, rather than the pseudo SU(3) quantum numbers $(\tilde{\lambda} \tilde{\mu})$.

The more detailed calculations of sect. 4 show that admixtures of $\tilde{L}=J \mp \frac{1}{2}$ into 
ground states with $\tilde{L}=J \pm \frac{1}{2}$ within the leading pseudo $\mathrm{SU}(3)$ representations must be expected to make contributions to $\mu$ of the order of $0.2 \mathrm{n} . \mathrm{m}$. Core contributions must also be expected to be of order $0.2 \mathrm{n} . \mathrm{m}$. Nevertheless, the numbers of tables 9 and 10 seem to indicate that the simple approximate magnetic moment formula, eq. (20), gives a remarkably good zeroth approximation for the magnetic moments of heavy deformed nuclei.

\section{Spectra. The Eu and Tm Isotopes}

\subsection{ENERGY CALCULATIONS}

Since precise energy calculations for the full proton and neutron configurations of heavy deformed nuclei would involve a prohibitively complicated configuration mixing problem, it may be interesting to examine the results of a highly simplified shell-model calculation for odd- $Z$ nuclei, based solely on the leading pseudo $S U(3)$ representation for the natural-parity part of the proton configuration, to see whether the diagonalization of an effective interaction in this highly truncated subspace of the full shell-model space can at least predict the correct order of the $K$-bands. An interesting test for the pseudo SU(3) model can be found in the cases $n=7$ and $n=11$, (seven or eleven active positive-parity protons), in the pseudo $\tilde{f}-\tilde{p}$ shell, which can be expected to apply to the spectra of the ${ }_{63} \mathrm{Eu}$ and ${ }_{69} \mathrm{Tm}$ isotopes. In both of these cases the leading pseudo $S U(3)$ representation (table 5) has $(\lambda \tilde{\mu})=(11,2)$, with $\widetilde{K}_{L}=0$ and 2 ; yet the experimental observations would lead us to expect that the $\widetilde{K}_{L}=2$ band, split into $K_{J}=\frac{5}{2}$ and $\frac{3}{2}$ rotational bands, should be the predicted ground state band for the Eu isotopes $(n=7)$, while the $\tilde{K}_{L}=0$ band, with $K_{J}=\frac{1}{2}$, should be the predicted ground state band for the Tm isotopes $(n=11)$. For this reason we have diagonalized a simple effective interaction in the subspace of the leading pseudo $\mathrm{SU}(3)$ representation $(11,2)$ for the two cases, $n=7$ and $n=11$. Since the surface delta interaction (SDI) has proved to be a remarkably good effective interaction for highly truncated subspaces of the shell-model space, it has been chosen for the twobody part of the effective interaction. Since it is a scalar interaction in the pseudo orbital and pseudo spin spaces, it is at best a simplified form of more realistic effective interactions. However, the states of the leading pseudo SU(3) representations for odd $n$ nuclei also have $\bar{S}-\frac{1}{2}$, so that tensor terms (rank 2 in the pseudo orbital and spin spaces) would have zero matrix elements in any case; and the effect of vector (rank 1) terms can perhaps be partly absorbed by the one-body pseudo spin-orbit coupling term which, together with a one-body $\tilde{l}^{2}$ term, is needed to reproduce the singleparticle spectrum. The SU(3) irreducible tensor character of the SDI (table 7) shows that its major components have $\left(\tilde{\lambda}_{0} \tilde{\mu}_{0}\right)=(00)$ and $(22)$, with $\left(\tilde{\lambda}_{0} \tilde{\mu}_{0}\right)=(44)$ next in importance, a property which it shares with more realistic interactions in both the real $^{25}$ ) and pseudo orbital spaces. The effective Hamiltonian has therefore been 
chosen to have the simple form

$$
H=A \sum_{i=1}^{n} \tilde{l}_{i} \cdot \tilde{s}_{i}+B \sum_{i=1}^{n} \tilde{l}_{i}^{2}+H_{\mathrm{SDI}}(G)
$$

where the coefficients $A$ and $B$ of the one-body pseudo $\boldsymbol{l} \cdot \tilde{s}$ and $\boldsymbol{l}^{2}$ parts of the Hamiltonian have been chosen to reproduce as nearly as possible the single-particle spectrum of the $1 \operatorname{g}_{\frac{3}{4}} 2 \mathrm{~d}_{\frac{2}{2}} 2 \mathrm{~d}_{\frac{3}{2}} 3 \mathrm{~s}_{\frac{1}{4}}$ proton shell. The strengths have been chosen as follows

$$
A=-0.250 \mathrm{MeV}, \quad B=-0.225 \mathrm{MeV} \text {. }
$$

This leads to a $1 \mathrm{~g}_{\frac{2}{2}}-2 \mathrm{~d}_{\frac{5}{2}}$ splitting of $0.875 \mathrm{MeV}$ [the experimental evidence ${ }^{26}$ ) indicates that this splitting is between 0.75 and $1 \mathrm{MeV}$ ], and a $2 \mathrm{~d}_{\frac{1}{2}}-3 \mathrm{~s}_{\frac{1}{2}}$ splitting of 0.375 $\mathrm{MeV}$ (again in qualitative agreement with the experimental expectations). The coefficient $B$ has been chosen to give a splitting of $2.25 \mathrm{MeV}$ between the centers of gravity of the $d_{\frac{3}{4}} s_{\frac{1}{2}}$ and $g_{\frac{7}{2}} d_{\frac{3}{2}}$ doublets. Extrapolation from the region of 82-neutron nuclei ${ }^{7,26}$ ) indicates that the strength parameter $G$ for the SDI should be expected to fall in the range $G=0.1 \mathrm{MeV}$ to $0.25 \mathrm{MeV}$; (the coefficients of table 7 are for unit strength factor, $G=1$ ).

The decomposition of the Hamiltonian, (21), into SU(3) irreducible tensor components has been given in sect. 2. Its matrix elements within the leading pseudo SU(3)

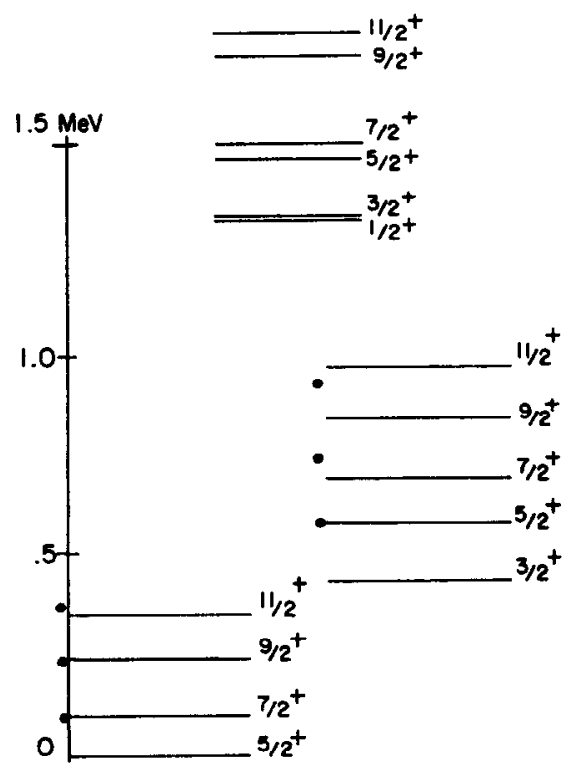

(a)

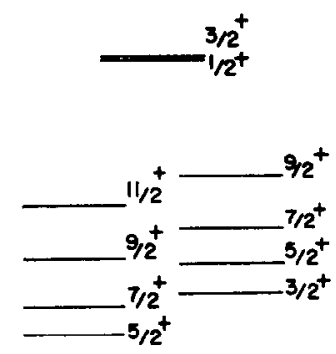

(b) $\quad{ }_{63}^{153} \mathrm{Eu}$

Fig. 3. The spectrum for $n=7$; seven active positive-parity protons coupled to leading pseudo SU(3) representation $(\tilde{\lambda} \tilde{\mu})=(11,2)$. (a) Eigenvalues of the Hamiltonian (21) with $A=-0.25, B=-0.225$ and $G=0.2 \mathrm{MeV}$. (b) The experimentally observed positive-parity levels of ${ }_{i 3}^{153} \mathrm{Eu}$. 


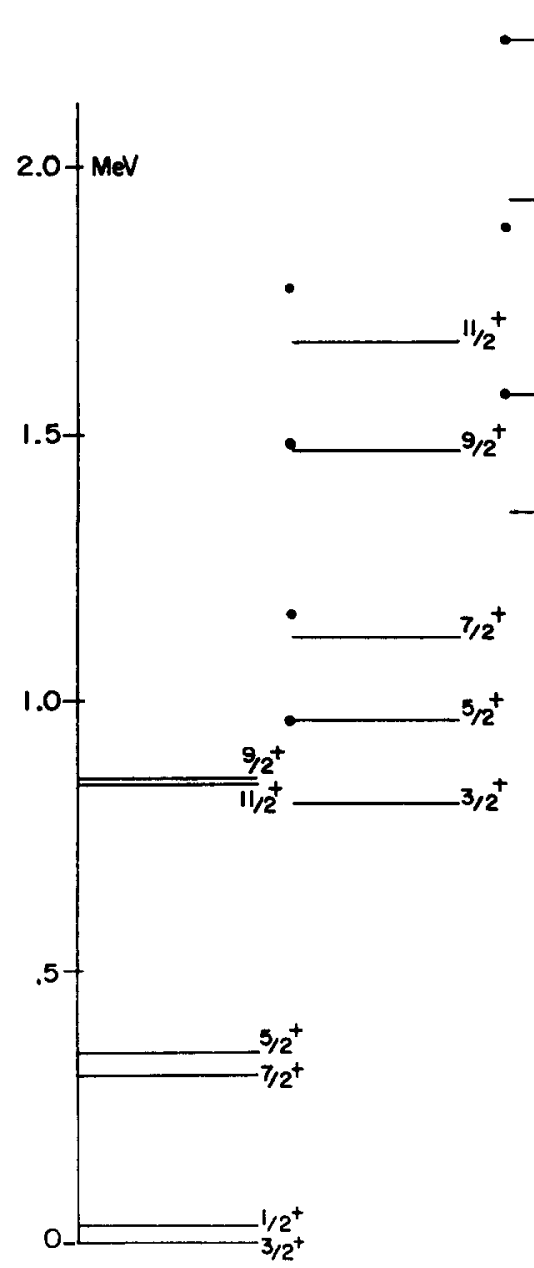

(a)

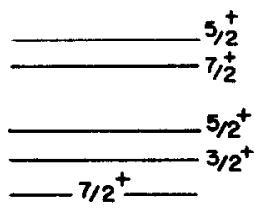

${ }_{69}^{171} \mathrm{Tm}$

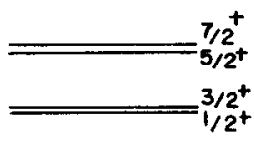

(b)

Fig. 4. The spectrum for $n=11$; eleven active positive-parity protons soupled to leading pseudo SU(3) representation $(\tilde{\lambda} \tilde{\mu})=(11,2)$. (a) Eigenvalues of the Hamiltonian (21) with $A=-0.25$, $B=-0.225, G=0.1 \mathrm{MeV}$. (b) The experimentally observed positive-parity levels of ${ }^{171} \mathrm{Tm}$; ref. ${ }^{31}$ ). (In ${ }_{69}^{169} \mathrm{Tm}$ the $\frac{9}{2}+, \frac{11}{2}^{+}$members of the $K=\frac{1}{2}$ ground state band are observed at 0.332 and $0.368 \mathrm{MeV}$, respectively.)

representation have been calculated with the use of eqs. (16) and (17) and with the coefficients of fractional parentage tabulated in ref. ${ }^{12}$ ). The qualitative nature of the results is insensitive to changes of the order of $\pm 0.1 \mathrm{MeV}$ in the parameters $A, B$ and $G$. The results for the two cases, $n=7$ and $n=11$, are shown in figs. 3 and 4 . We note that the two spectra are in qualitative agreement with the experimental spectra for the Eu and Tm isotopes. For $n=7$ (the Eu isotopes) it is the $\tilde{K}_{L}=2$ band, split into $K_{J}=\frac{5}{2}$ and $\frac{3}{2}$ bands, which lies lowest; while for $n=11$ (the Tm isotopes) 
TABLE 11

Eigenvectors for $n=7$

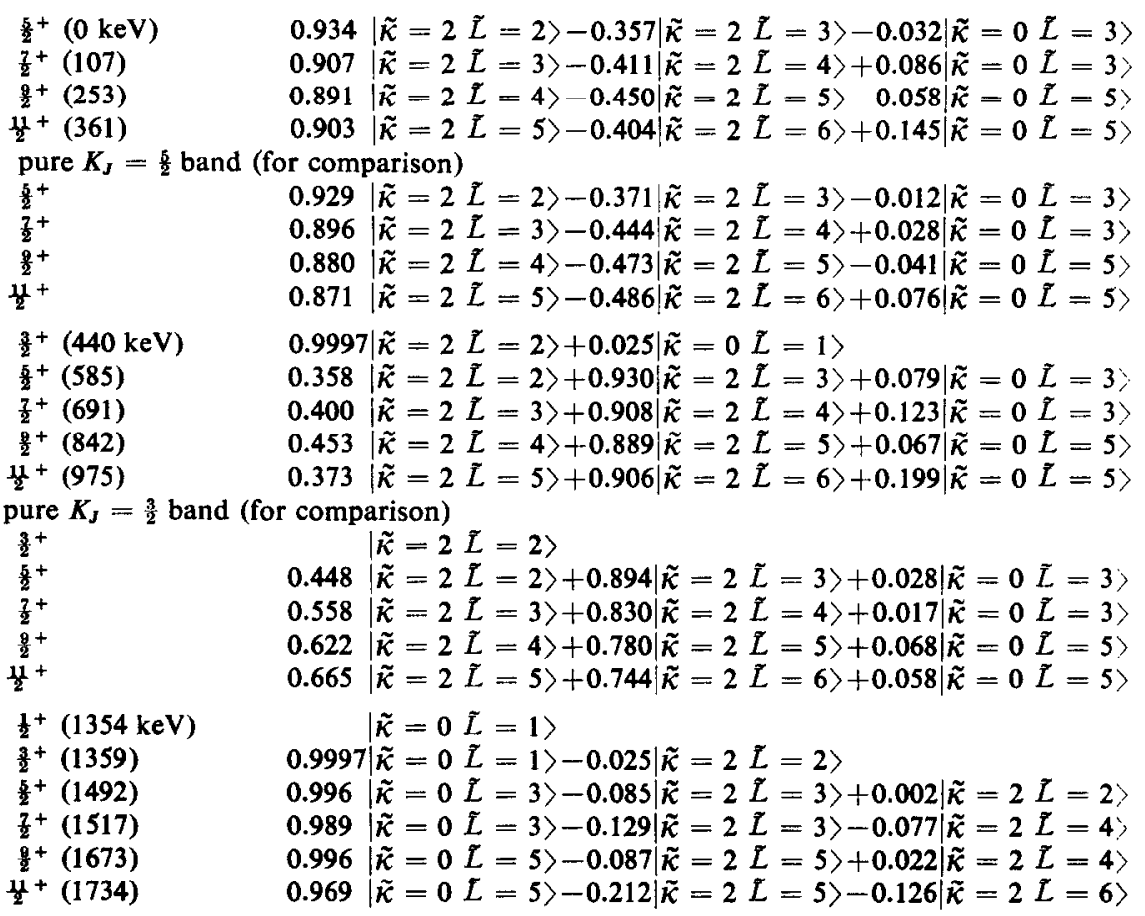

$|\tilde{\kappa} \tilde{L}\rangle$ is an abbreviation for $\left|(\tilde{\lambda} \tilde{\mu})=(11,2) \tilde{\kappa} \mathcal{L} S=\frac{1}{2} J\right\rangle$.

it is the $\widetilde{K}_{L}=0$ band, with $K_{J}=\frac{1}{2}$, which lies lowest. Moreover, the $K_{J}=\frac{1}{2}$ bands have the appearance of rotational bands with decoupling parameter values around $a=-1$, characteristic of this region; more specifically, for $n=11$ the predicted ground state $K_{J}=\frac{1}{2}$ band has the appearance of a band with decoupling parameter $a \approx-1.2$, while for $n=7$ the predicted $K_{J}=\frac{1}{2}$ band, at higher excitation, has the appearance of a rotational band with $a \approx-0.9$. The experimentally observed $K=\frac{1}{2}$ bands in both the $\mathrm{Eu}$ and $\mathrm{Tm}$ isotopes on the other hand correspond to decoupling parameters, $a \approx-0.9 ;\left(\frac{1}{2}^{+}\right.$states just below $\frac{3}{2}^{+}$states, $\left.\ldots\right)$. The spacings of the predicted $K=\frac{5}{2}$ and $\frac{3}{2}$ bands are nearly rotational; (a pure $I(I+1)$ spacing is indicated by the dots in figs. 3 and 4). The eigenvectors for the Hamiltonian (21) are shown in table 11 for the case $n=7$. We note that the ground state and first excited band are nearly pure $\tilde{\kappa}=2\left(\widetilde{K}_{L}=2\right)$ bands. Moreover, even the $K_{J}$ values of these bands are nearly pure, where $K_{J}=\widetilde{K}_{L}+\widetilde{K}_{s}$. The expansion of pure $K_{J}$ band state vectors in terms of state vectors $|(\tilde{\lambda} \tilde{\mu})=(11,2) \tilde{\kappa} \tilde{L} \tilde{S} J M\rangle$ are shown in table 11 for $K_{J}=\frac{s}{2}$ and $\frac{3}{2}$ for comparison with the eigenvectors of the model Hamiltonian (21). Note that $K_{J}$ is the projection quantum number $K$ for the real total angular momentum $J$, 
(hence no ). Here we have used the relation between the states $\left|(\lambda \mu) K_{J} S J M\right\rangle$ and the states $\left|(\lambda \mu) K_{L} L S J M\right\rangle$ given by Harvey, (see eqs. (3.45) and (H.12) of ref. $\left.{ }^{2}\right)$ ), with $L, S, K_{L}, K_{S}$ replaced by $\tilde{L}, \tilde{S}, \tilde{K}_{L}, \tilde{K}_{S}$ :

$$
\left|(\tilde{\lambda} \tilde{\mu}) K_{J} S J M\right\rangle=\sum_{\tilde{L}}\left|(\tilde{\lambda} \tilde{\mu}) \tilde{K}_{L} \tilde{L} \tilde{S} J M\right\rangle \frac{a\left(\tilde{\lambda} \tilde{\mu} \tilde{K_{L}} \tilde{L}\right)}{A\left(\tilde{\lambda} \tilde{\mu} K_{J} J\right)}\left\langle\tilde{L} \tilde{K}_{L} \tilde{S}=\frac{1}{2} \tilde{K}_{S} \mid J K_{J}\right\rangle,
$$

and we have made the transformation from Elliott state vectors $\left|(\tilde{\mu} \tilde{\mu}) \tilde{K}_{L} \tilde{L} \tilde{S} J M\right\rangle$, with label $\tilde{K}_{L}$, to the orthonormal basis $|(\tilde{\lambda} \tilde{\mu}) \tilde{\kappa} \tilde{L} \tilde{S} J M\rangle$, with the Vergados $\left.{ }^{15}\right)$ label $\tilde{\kappa}$, which has been used in the present calculations. Note that for $(\tilde{\lambda} \tilde{\mu})=(11,2)$ states with $\tilde{\kappa}=0$, and $\tilde{\kappa}=2 \tilde{L}=$ even, are identical with the Elliott state vectors with $\widetilde{K}_{L}=0$, and $\widetilde{K}_{L}=2 \tilde{L}=$ even. States with $\tilde{\kappa}=2$ and $\tilde{L}=$ odd can be related to the Elliott state vectors with $\widetilde{K}_{L}=2$ and 0 through the transformation coefficients of table $2 \mathrm{~B}$ of ref. ${ }^{15}$ ). For example

$$
\begin{array}{r}
|(\tilde{\lambda} \tilde{\mu})=(11,2) \tilde{\kappa}=2 \tilde{L}=3\rangle=1.00048\left|(\tilde{\lambda} \tilde{\mu})=(11,2) \tilde{K}_{L}=2 \tilde{L}=3\right\rangle \\
-0.03114\left|(\tilde{\lambda} \tilde{\mu})=(11,2) \tilde{K}_{L}=0 \tilde{L}=3\right\rangle, \\
|(\tilde{\lambda} \tilde{\mu})=(11,2) \tilde{\kappa}=2 \tilde{L}=5\rangle=1.00379\left|(\tilde{\lambda} \tilde{\mu})=(11,2) \tilde{K}_{L}=2 \tilde{L}=5\right\rangle \\
-0.08713\left|(\tilde{\lambda} \tilde{\mu})=(11,2) \tilde{K}_{L}=0 \tilde{L}=5\right\rangle .
\end{array}
$$

The diagonalization of the simple effective Hamiltonian (21) within the basis spanned by the state vectors of the leading pseudo SU(3) representation for $n=7$ thus leads to a spectrum of rotational bands of nearly good $K_{J}$, where the bands with $K_{J}=\frac{5}{2}, \frac{3}{2}$, and $\frac{1}{2}$ appear in the right order and the level spacings within the bands are also in qualitative agreement with the experimental observations. Fig. 3 shows the experimental spectrum of ${ }_{63}^{153} \mathrm{Eu}$ alongside the spectrum predicted for $n=7$ with the parameters $A=-0.25, B=-0.225$ and $G=0.2 \mathrm{MeV}$. We would certainly not expect good quantitative agreement between the model predictions and experiment. And indeed, the theoretical rotational constants are somewhat too large. For example, a $\frac{7}{2}{ }^{+}-\frac{5}{2}+$ spacing of $107 \mathrm{keV}$ is predicted for the members of the $K_{J}=\frac{5}{2}$ band compared with an observed spacing of $83 \mathrm{keV}$ in ${ }^{153} \mathrm{Eu}$ and $75 \mathrm{keV}$ in ${ }^{155} \mathrm{Eu}$. The spacing of levels within a band is governed largely by the SDI strength parameter $G$. (With $G=0.1 \mathrm{MeV}$, e.g., the predicted $\frac{7}{2}^{+}-\frac{5}{2}^{+}$spacing of the $K_{J}=\frac{5}{2}$ band is reduced to $52 \mathrm{keV}$.) The predicted separation of the $K_{J}=\frac{3}{2}$ and $\frac{5}{2}$ bands is also somewhat large, $440 \mathrm{keV}$ between the $\frac{3}{2}^{+}$and $\frac{5}{2}^{+}$band heads, compared with an experimental separation of $103 \mathrm{keV}$ in ${ }^{153} \mathrm{Eu}$ and $246 \mathrm{keV}$ in ${ }^{155} \mathrm{Eu}$, where this particular spacing is quite insensitive to changes in the parameter $G$ but is a sensitive function of the spin-orbit strength parameter $A$. (Since our highly simplified model calculation has ignored $\mathrm{n}$-p interactions altogether, it can of course not account for differences of the order of $150 \mathrm{keV}$ between the spectra of ${ }^{153} \mathrm{Eu}$ and ${ }^{155} \mathrm{Eu}$ and should not be expected to make quantitative predictions for the $K_{J}=\frac{3}{2}$ and $\frac{5}{2}$ band separations.) Finally, the separation between the $K_{J}=\frac{1}{2}$ band and the $K_{J}=\frac{5}{2}, \frac{3}{2}$ bands is governed largely by the strength parameter $B$ of the one-body $\tilde{l}^{2}$ term in the model Hamiltonian. The predict- 
ed separation of $1354 \mathrm{keV}$ is again too large compared with an experimental separation ${ }^{27}$ ) of $700 \mathrm{keV}$ in ${ }^{153} \mathrm{Eu}$ and $910 \mathrm{keV}$ in ${ }^{155} \mathrm{Eu}$. [Experimentally, there are indications ${ }^{27}$ ) that an observed intrinsic excitation with $K_{J}=\frac{1}{2}$, corresponding to our predicted $K_{J}=\frac{1}{2}$ band, lies very close to a second $K=\frac{1}{2}$ band, also with $a \approx-0.9$, which may arise from $\gamma$-vibration coupled to the ground state rotational band.]

It is interesting to note that it is mainly the sign of the parameter $B$ which determines the relative positions of the $\widetilde{K}_{L}=0$ bands, with $K_{J}=\frac{1}{2}$, and the $\tilde{K}_{L}=2$ bands, $\left(K_{J}=\frac{5}{2}, \frac{3}{2}\right)$, in the Tm isotopes $(n=11)$ as contrasted with the Eu isotopes $(n=7)$. With a large positive value of $B$, the $K_{J}=\frac{1}{2}$ band would lie above the $K_{J}=\frac{5}{2}$, $\frac{3}{2}$ bands in $\mathrm{Tm}$ while it would lie below the $K_{J}=\frac{5}{2}, \frac{3}{2}$ bands in Eu. However, the negative value of $B$ is required by the experimental fact that the $l=3$ doublet, $\left(\lg _{\frac{2}{2}} 2 \mathrm{~d}_{\frac{1}{2}}\right)$, lies considerably below the $l=1$ doublet, $\left(2 \mathrm{~d}_{\frac{1}{3}} 3 \mathrm{~s}_{\frac{1}{2}}\right)$, in the proton spectrum of the rareearth nuclei. Further, it is interesting to note that this order of the $l=3$ and $l=1$ levels is one which tends to destroy the goodness of the $\mathrm{SU}(3)$ quantum numbers for nuclei with $N \approx Z$ in the beginning of the real $\mathrm{f}-\mathrm{p}$ shell ${ }^{28}$ ). It is not clear whether a corresponding argument can be carried over to the pseudo $\tilde{\mathrm{f}}-\tilde{\mathrm{p}}$ shell of strongly deformed nuclei. For the present we are content to test the consequences of the pseudo SU(3) model and base its possible validity on the qualitative arguments presented in sect. 1; (these may depend very much on the mediating influence of the filled neutron shells and the nucleons in the core).

TABLE 12

Eigenvectors for $n=11$

\begin{tabular}{|c|c|}
\hline$\frac{3}{2}^{+}(0 \mathrm{keV})$ & $0.9996|\tilde{\kappa}=0 \tilde{L}=1\rangle-0.029|\tilde{\kappa}=2 \tilde{L}=2\rangle$ \\
\hline$\frac{1^{+}}{2}(30)$ & $|\tilde{\kappa}-0 I-1\rangle$ \\
\hline $\begin{array}{l}\frac{7}{2}+(311) \\
\frac{5}{2}+(356)\end{array}$ & $\begin{array}{l}0.998|\tilde{\kappa}=0 I=3\rangle+0.050|\tilde{\kappa}=2 L=3\rangle-0.029|\tilde{\kappa}=2 \tilde{L}=4\rangle \\
0.981|\tilde{\kappa}=0 L=3\rangle+0.184|\tilde{\kappa}=2 L=3\rangle+0.064|\tilde{\kappa}=2 L=2\rangle\end{array}$ \\
\hline $\begin{array}{l}\frac{1}{2}+(849) \\
\frac{9}{2}+(858)\end{array}$ & $\begin{array}{l}0.984|\tilde{\kappa}=0 \tilde{L}=5\rangle+0.179|\tilde{\kappa}=2 \tilde{L}=5\rangle-0.010|\tilde{\kappa}=2 \tilde{L}=6\rangle \\
0.915|\tilde{\kappa}=0 \tilde{L}=5\rangle+0.355|\tilde{\kappa}=2 \tilde{L}=5\rangle+0.191|\tilde{\kappa}=2 \tilde{L}=4\rangle\end{array}$ \\
\hline $\begin{array}{l}\frac{3}{2}+(818) \\
\frac{5}{2}+(969) \\
\frac{7}{2}+(1126) \\
\frac{e^{+}}{2}+(1441) \\
\frac{1+}{2}+(1678)\end{array}$ & $\begin{array}{l}0.9996|\tilde{\kappa}=2 \tilde{L}=2\rangle+0.029|\tilde{\kappa}=0 \tilde{L}=1\rangle \\
0.613|\tilde{\kappa}=2 \tilde{L}=2\rangle+0.768|\tilde{\kappa}=2 \tilde{L}=3\rangle-0.184|\tilde{\kappa}=0 \tilde{L}=3\rangle \\
0.741|\tilde{\kappa}=2 \tilde{L}=3\rangle+0.672|\tilde{\kappa}=2 \tilde{L}=4\rangle-0.018|\tilde{\kappa}=0 \tilde{L}=3\rangle \\
0.865|\tilde{\kappa}=2 \tilde{L}=4\rangle+0.380|\tilde{\kappa}=2 \tilde{L}=5\rangle-0.328|\tilde{\kappa}=0 \tilde{L}=5\rangle \\
0.835|\tilde{\kappa}=2 \tilde{L}=5\rangle+0.530|\tilde{\kappa}=2 \tilde{L}=6\rangle-0.147|\tilde{\kappa}=0 \tilde{L}=5\rangle\end{array}$ \\
\hline $\begin{array}{r}\frac{5}{2}+(1357) \\
\frac{7}{2}+(1584) \\
\frac{9}{2}+(1933) \\
\frac{11}{2}+(2235)\end{array}$ & 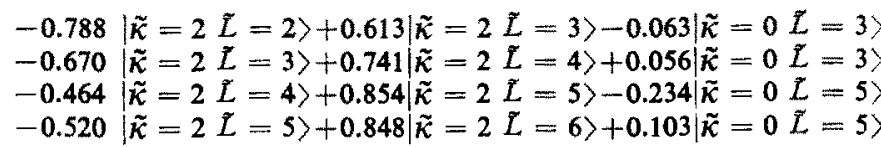 \\
\hline
\end{tabular}

$|\tilde{\kappa} \tilde{L}\rangle$ is an abbreviation for $\left|(\tilde{\lambda} \tilde{\mu})=(11,2) \tilde{\kappa} \tilde{L} \tilde{S}=\frac{1}{3} J\right\rangle$.

To illustrate the sensitivity of the expansion coefficients to changes in the parameters $A, B, G$, we note that the coefficients of the second ${ }^{+}{ }^{+}$state, which are

$0.613,0.768,-0.184$ for $A=-0.25, B=-0.225, G=0.1 \mathrm{MeV}$ above, change to

$0.655,0.733,-0.182$ for $A=-0.25, B=-0.225, G=0.2 \mathrm{MeV}$, and to

$0.586,0.770,-0.251$ for $A=-0.35, B=-0.225, G=0.2 \mathrm{MeV}$, and to

$0.732,0.669,-0.127$ for $A=-0.25, B=-0.35, G=0.2 \mathrm{MeV}$ 
Fig. 4 shows the experimentally observed positive-parity levels of ${ }_{69}^{171} \mathrm{Tm}$ alongside the spectrum predicted for $n=11$ with parameters $A=-0.25, B=-0.225$ and $G=0.1 \mathrm{MeV}$. The eigenvectors for this case are shown in table 12 . The ground state band is almost a pure $K_{J}=\frac{1}{2}(\tilde{\kappa}=0)$ band. Its predicted rotational spacings are again too large; the $\frac{5}{2}^{+}$member of the band which is observed at $\approx 117 \mathrm{keV}$ in ${ }^{167} \mathrm{Tm}$, ${ }^{169} \mathrm{Tm}$ and ${ }^{171} \mathrm{Tm}$ is predicted at $356 \mathrm{keV}$ (with $G=0.2 \mathrm{MeV}$ it would lie even higher at $431 \mathrm{keV}$ ). The first positive-parity intrinsic excitation in ${ }^{171} \mathrm{Tm}$ gives rise to a $K=\frac{7}{2}$ rotational band, described in the unified model by an excitation into the Nilsson orbit $[404]_{2}^{\frac{7}{2}}{ }^{+}$. In the pseudo SU(3) model this would correspond to an excitation out of the leading pseudo $\mathrm{SU}(3)$ representation into one of the representations with $2 \tilde{\lambda}+\tilde{\mu}=$ 21 , which for $n=11$ includes the possibilities $(\lambda \tilde{\mu})=(93),(85)$ and $(77)$ or mixtures of these. (The ground state magnetic moment indicates that admixtures of lower representations may be important in the $\mathrm{Tm}$ isotopes). The next observed positiveparity band in ${ }^{171} \mathrm{Tm}$ has $K=\frac{3}{2}$. It is interesting to note that the $\tilde{\kappa}=2$ band of the leading representation $(11,2)$ is split into $K_{J}=\frac{3}{2}$ and $K_{J}=\frac{5}{2}$ bands, where in contrast to the Eu isotopes, it is the $\frac{3}{2}$ band which lies lower. The eigenvectors of table 12 also show that the $K_{J}$ values of these bands are not as pure as in the case of the Eu isotopes.

Finally, we note that the predicted level schemes of the $\mathrm{Eu}$ and $\mathrm{Tm}$ isotopes have the appearance of spectra which are connected by a particle-hole relationship. To understand these similarities and differences in the spectra for the two cases, $n=7$ and $n=11$, it should be noted that these do indeed correspond to the case of "one particle" and "one hole", respectively, in the subspace of pseudo SU(3) representations with a maximum value of $2 \tilde{\lambda}+\tilde{\mu}=24$ for the $\tilde{N}=3$ shell. For $6 \leqq n \leqq 12$ all leading pseudo $S U(3)$ representations have $2 \tilde{\lambda}+\tilde{\mu}=24$; in particular for $n=6$ and $n=12$, the leading pseudo $\mathrm{SU}(3)$ representations have $(\tilde{\lambda} \tilde{\mu})=(12,0)$.

\subsection{SPECTROSCOPIC FACTORS}

Other predictions of the simple pseudo SU(3) model can be compared with experimental observations. Spectroscopic factors for single-proton transfer reactions have a simple form under the assumption that the proton configurations in both target and final nuclei are coupled to leading pseudo SU(3) representation. For the reactions

${ }_{62}^{A} \mathrm{Sm}\left({ }^{3} \mathrm{He}, \mathrm{d}\right){ }_{63}^{A+1} \mathrm{Eu}$ to positive-parity states in the Eu isotopes, spectroscopic factors would then be given by the reduced matrix element of a proton creation operator between the configuration with $n=6$ coupled to $(\tilde{\lambda} \tilde{\mu})=(12,0)$ and $n=7$ coupled to $(\tilde{\lambda} \tilde{\mu})=(11,2)$. Specifically,

$$
\begin{aligned}
\mathscr{S}=\mid \sum_{\tilde{\kappa}} c_{\tilde{\kappa} \tilde{L}}\langle(12,0) \tilde{L}=0 ;(30) \tilde{l}=\tilde{L} \| & \|(11,2) \tilde{\kappa} \tilde{L}\rangle \\
& \times\left.\left\langle(11,2) \tilde{S}=\frac{1}{2}\left\||| a_{(30) \frac{1}{2}}^{\dagger}\right\|(12,0) \tilde{S}=0\right\rangle\right|^{2},
\end{aligned}
$$

with $I=\tilde{L}=3$ only for transfers of $\mathrm{g}_{\frac{7}{2}}$ and $\mathrm{d}_{\frac{3}{2}}$ protons to final states with $J=\frac{7}{2}$ and $\frac{5}{2}$, and with $l=\tilde{L}=1$ only for transfers of $\mathrm{d}_{\frac{1}{2}}$ and $\mathrm{s}_{\frac{1}{2}}$ protons to final states with 
TABLE 13

Pseudo SU(3) model predictions for the spectroscopic factors for the reactions ${ }_{62}{ }^{A} \mathrm{Sm}\left({ }^{3} \mathrm{He}, \mathrm{d}\right)_{63}{ }^{A+1} \mathrm{Eu}$

\begin{tabular}{|c|c|c|}
\hline$K_{J}$ & $I$ & $\mathscr{S}$ \\
\hline \multirow[t]{2}{*}{$K_{J}=\frac{5}{2}$} & $2^{+}$ & 0.05 \\
\hline & $\frac{7}{2}+$ & 0.30 \\
\hline \multirow[t]{3}{*}{$K_{\mathrm{J}}=\frac{3}{2}$} & $\frac{3_{2}^{2}}{2}$ & 0.0001 \\
\hline & $\frac{5}{2}{ }^{+}$ & 0.32 \\
\hline & $\frac{7}{2}+$ & 0.05 \\
\hline \multirow[t]{4}{*}{$K_{J}=\frac{1}{2}$} & $\frac{1}{2}^{+}$ & 0.23 \\
\hline & $\frac{3}{2}+$ & 0.23 \\
\hline & $\frac{5}{2}+$ & 0.12 \\
\hline & $\frac{7}{2}+$ & 0.14 \\
\hline
\end{tabular}

$J=\frac{3}{2}$ and $\frac{1}{2}$. (Note that the model predicts $\mathscr{S}=0$ for final states with $J \geqq \frac{9}{2}$.) In this case the triple-barred reduced matrix element has the value $\left(\frac{91}{66}\right)^{\frac{1}{2}}$; the doublebarred coefficient is the appropriate $\mathrm{SU}(3) \supset \mathrm{R}(3)$ Wigner coefficient; and the coefficients $c_{\kappa \tilde{L}}$ are the expansion coefficients for the state vectors for $n=7$ which can be read from table 11. Numerical values for these spectroscopic factors are given in table 13. Levels in ${ }^{153} \mathrm{Eu}$ and ${ }^{155} \mathrm{Eu}$ have recently been studied by the $\left({ }^{3} \mathrm{He}, \mathrm{d}\right)$ reaction by Ungrin et al. ${ }^{27}$ ). Spectroscopic factors have not been evaluated; but the magnitudes of the observed cross sections are, possibly with one exception, in qualitative agreement with the predictions of table 13. By far the strongest observed cross sections involve the transitions to the $\frac{5}{2}^{+}$member of the $K=\frac{3}{2}$ band and the unresolved $\frac{1}{2}^{+}$and $\frac{3}{2}^{+}$members of the $K=\frac{1}{2}$ band. However, the predicted spectroscopic factor to the $\frac{7}{2}^{+}$member of the $K=\frac{5}{2}$ band is of the same strength as that for the $\frac{5}{2}^{+}$member of the $K=\frac{3}{2}$ band. [DWBA calculations ${ }^{33}$ ), with the optical parameters of ref. ${ }^{27}$ ) would give relative peak cross sections of approximately $1: 4$ for these two states using the spectroscopic factors of table 13, whereas the observed peak cross sections differ by an order of magnitude. The observed angular distribution for the unresolved $\frac{1}{2}^{+} \frac{3}{2}^{+}$doublet of the $K=\frac{1}{2}$ band is not in disagreement with an equal mixture of $l=0$ and $l=2$ transfer ${ }^{33}$ ).]

\subsection{THE M1 TRANSITION PROBABILITIES}

In view of the seeming success of the pseudo SU(3) model in predicting the magnetic moments of deformed nuclei, it is also interesting to examine the M1 transition probabilities between the states of the leading pseudo SU(3) representations. Since $L$ is not a good quantum number for the eigenvectors of the simple model Hamiltonian (21), even the predictions for the ground state magnetic moments within the leading pseudo SU(3) representations are modified somewhat over the simple predictions of eq. (20). For the $(11,2)$ representation of $n=7$, for example, the predicted $\frac{5}{2}^{+}$ground state (table 11 ) has a $12.8 \%$ admixture of $\tilde{L}=3$. This admixture of $\tilde{L}=3$ into a state with a predominant component of $\tilde{L}=2$ changes the prediction for the ground state magnetic moment from $\mu=1.73$ (for pure $\tilde{L}=2$ ) to a value of 1.90 n.m. for 
the predicted ground state of table 11. These numbers are to be compared with the experimental value $\mu=1.53$ for ${ }^{153} \mathrm{Eu}$. In ${ }^{153} \mathrm{Eu}$ some excited state magnetic moments have also been measured. The lowest $\frac{3}{2}^{+}$excited state is an almost pure $\tilde{L}=2$ state with a predicted magnetic moment (using eq. (20)) of $1.95 \mathrm{n} . \mathrm{m}$. in good agreement with the experimental value ${ }^{24}$ ) of $2.0 \mathrm{n}$.m. for the $\frac{3}{2}^{+}$state at $103 \mathrm{keV}$ in ${ }^{153} \mathrm{Eu}$. On the other hand, the predicted value for the magnetic moment of the lowest $\frac{7}{2}^{+}$ excited state is $2.95 \mathrm{n} . \mathrm{m}$. for the state vector of table 11 (compared with a predicted value of $2.73 \mathrm{n} . \mathrm{m}$. for a pure $\tilde{L}=3$ state.) Neither of these is in agreement with the experimental value ${ }^{29}$ ) of $1.8 \mathrm{n} . \mathrm{m}$. for the $\frac{7}{2}^{+}$state at $83 \mathrm{keV}$ in ${ }^{153} \mathrm{Eu}$.

To calculate the M1 transition probabilities we need the matrix elements of the magnetic moment operator (see eq. (7)) between state vectors such as those of table 11. The complicated tensor part of the magnetic moment operator (the last term of eq. (7)) again makes very small contributions to such matrix elements. (Its contributions to matrix elements off-diagonal in $\tilde{\kappa}, \tilde{L}$, or $J$ differ from its contribution to the diagonal matrix elements only by trivial angular momentum recoupling coefficients and the $\tilde{\kappa}, \tilde{L}$ dependent $\mathrm{SU}(3) \supset \mathrm{R}(3)$ Wigner coefficients, see eq. (16)). For example, it makes a contribution of only -0.0038 n.m. to the matrix element connecting a component with $\tilde{\kappa}=2, \tilde{L}=2, \tilde{S}=\frac{1}{2}, J=M=\frac{3}{2}$ to a component with $\tilde{\kappa}=2$, $\tilde{L}=3, \tilde{S}=\frac{1}{2}, J=M=\frac{5}{2}$ in the $(11,2)$ representation for $n=7$. It is therefore again a very good approximation to neglect this complicated tensor term altogether and calculate matrix elements of the magnetic moment operator between state vectors within the leading pseudo SU(3) representations with the simple expression

$$
\begin{aligned}
& \left\langle(\tilde{\lambda} \tilde{\mu}) \beta^{\prime} J^{\prime} M^{\prime}\left|\mu_{q}\right|(\tilde{\lambda} \tilde{\mu}) \beta J M\right\rangle=\left[\frac{2 J+1}{2 J^{\prime}+1}\right]^{\frac{1}{2}}\left\langle J M 1 q \mid J^{\prime} M^{\prime}\right\rangle \sum_{\tilde{\kappa} \tilde{L^{\prime}} \tilde{L}^{\prime}} c_{\tilde{\kappa} \tilde{L}}^{(\beta)} c_{\tilde{\kappa}^{\prime} \tilde{L}^{\prime}}^{\left.(\beta)^{\prime}\right)}\left\{g_{[}[J(J+1)]^{\frac{1}{2}}\right. \\
& \left.\times \delta_{J J^{\prime}} \delta_{\tilde{\kappa} \tilde{\kappa}^{\prime}} \delta_{\tilde{L} \tilde{L}^{\prime}}-\frac{1}{3}\left(g_{s}-g_{l}\right)\left[\frac{3\left(2 J^{\prime}+1\right)}{2}\right]^{\frac{1}{2}}\left\{\begin{array}{ccc}
\frac{1}{2} & \tilde{L} & J \\
J^{\prime} & 1 & \frac{1}{2}
\end{array}\right\}(-1)^{\tilde{L}+\frac{1}{2}+J^{\prime}+1} \delta_{\tilde{\kappa} \tilde{\kappa}^{\prime}} \delta_{\tilde{L} \tilde{L}^{\prime}}\right\}
\end{aligned}
$$

where the coefficients $c_{\kappa \tilde{L}}^{(\beta)}$ are the expansion coefficients for the eigenvectors of the model Hamiltonian (21) in terms of the state vectors $\left|(\tilde{\lambda} \tilde{\mu}) \tilde{\kappa} \tilde{L} \tilde{S}=\frac{1}{2} J M\right\rangle$. For $n=7$ these can be read from table 11. Also, for $n=7$ the general label $\beta$, identifying the state, could be replaced by the label $K_{J}$. The $B(\mathrm{M} 1)$ values have been calculated with eq. (25) and the expansion coefficients of table 11 for the transitions among the various members of the $K_{J}=\frac{5}{2}$ and $K_{J}=\frac{3}{2}$ bands for the Eu isotopes. The predicted $B(\mathrm{M} 1)$ values are shown in units of $(3 / 4 \pi)(c \hbar / 2 M c)^{2}$ in fig. 5 and compared with the experimentally observed $\left.{ }^{30}\right) B(\mathrm{M} 1)$ values for ${ }^{153} \mathrm{Eu}$. The relative magnitudes of the predicted intraband transitions $K_{J}=\frac{3}{2} \rightarrow \frac{3}{2}$ or $K_{J}=\frac{5}{2} \rightarrow \frac{5}{2}$ are in qualitative agreement with the Alaga rule; that is, they are approximately proportional to $\left\langle J K_{J} 10\right|$ $\left|J^{\prime} K_{J}\right\rangle^{2}$. The predicted $\mathrm{Ml}$ transition probability for the intraband transition $\frac{5}{2}^{+} \rightarrow \frac{3}{2}^{+}\left(K_{J}=\frac{3}{2} \rightarrow \frac{3}{2}\right)$ is too small by a factor of about 4 compared with its experimental value; that is the corresponding matrix element is too small by a factor of $\approx 2$. In view of the extreme zeroth-order nature of our calculation and the simplicity of 


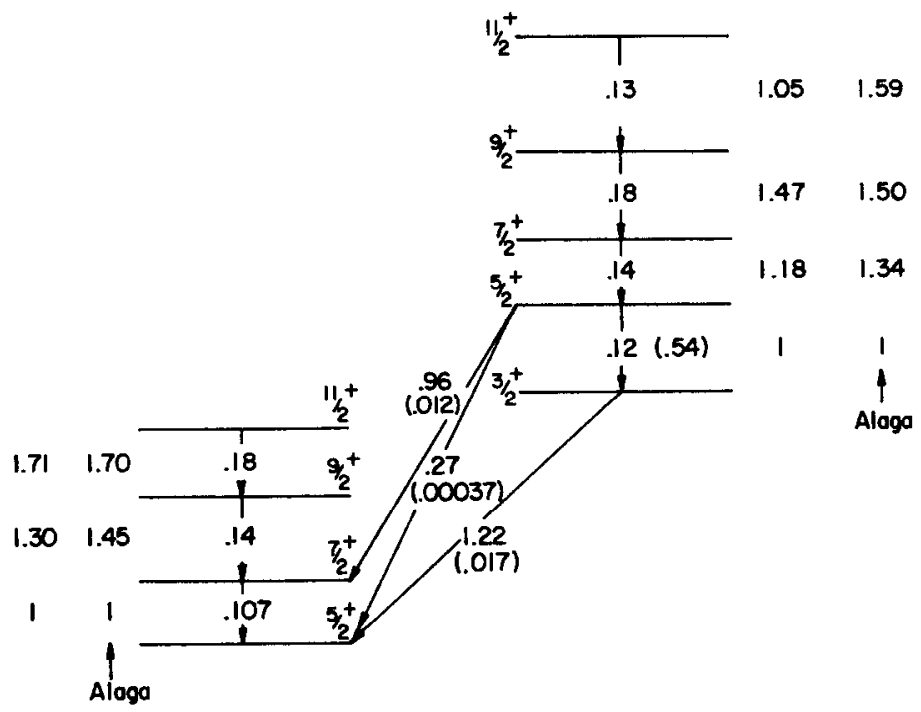

Fig. 5. The $B(\mathrm{M} 1)$ values in units of $(3 / 4 \pi)(e \hbar / 2 M c)^{2}$ for the $K_{J}=\frac{5}{2}$ and $\frac{3}{2}$ bands for $n=7$. The numbers in parentheses are for the experimentally observed $B(\mathrm{M} 1)$ values for ${ }_{63}^{153} \mathrm{Eu}$ [ref. ${ }^{30}$ )]. All other numbers are for the predicted spectrum of fig. 3 and table 11. The predicted relative $B(M 1)$ values for the intraband transitions are also compared with relative $B(M 1)$ values given by the Alaga rule.

our model this may be considered qualitative agreement between the predicted and experimental values. However, the situation is very different for the interband M1 transition probabilities, $\left(K_{J}=\frac{3}{2} \rightarrow K_{J}=\frac{5}{2}\right)$. The experimentally observed interband M1 transition probabilities are strongly hindered ${ }^{30}$ ), whereas the predicted interband $B(\mathrm{M} 1)$ values are large compared with the intraband values. Both the $\frac{3}{2}^{+}$member of the $K_{J}=\frac{3}{2}$ band and the $\frac{5}{2}^{+}$member of the $K_{J}=\frac{5}{2}$ band have predominant components with $\tilde{\kappa}=2, \tilde{L}=2$ (table 11 ), leading to a large M1 matrix element for the transition $\frac{3}{2}^{+} \rightarrow \frac{5}{2}^{+}\left(K_{J}=\frac{3}{2} \rightarrow K_{J}=\frac{5}{2}\right)$. Essentially the only hindrance factor in the pseudo $\mathrm{SU}(3)$ description for this transition comes from the square of the factor $-\frac{1}{3}$ which multiplies the $\left(g_{s}-g_{t}\right)$ value in the $\bar{S}$ term for the magnetic moment operator, eq. (7). (The contribution of the complicated tensor term of $\mu$ to the matrix element connecting $\tilde{\kappa}=2, \tilde{L}=2, J=\frac{3}{2}$ to $\tilde{\kappa}=2, \tilde{L}=2, J=\frac{5}{2}$ is much too small to make a significant contribution to the hindrance factor, and in fact has the wrong sign.) In Weisskopf units, (see eq. (16) of the appendix), the hindrance factor for the transition $\frac{3}{2}^{+} \rightarrow \frac{5}{2}^{+}\left(K_{J}=\frac{3}{2} \rightarrow K_{J}=\frac{5}{2}\right)$ has a predicted value of 6 compared with experimentally observed ${ }^{30}$ ) hindrance factors of 430 in ${ }^{153} \mathrm{Eu}$ and 3400 in ${ }^{155} \mathrm{Eu}$, an order of magnitude failure for our model. This is a serious stumbling block for the pseudo $\mathrm{SU}(3)$ model, since this prediction is not merely characteristic of the case $n=7$ of the Eu isotopes. Predicted M1 transition probabilities for interband transitions $K_{J}=\tilde{\kappa}-\frac{1}{2} \rightarrow K_{J}^{\prime}=\tilde{\kappa}+\frac{1}{2}$ within the leading pseudo $\mathrm{SU}(3)$ representations 
can be expected to be quite strong for all values of $n$. In the Bohr-Mottelson rotational model on the other hand the corresponding interband transitions can always be expected to be strongly hindered. In the limit of large deformations and asymptotic quantum numbers $\left[\mathrm{Nn}_{z} \Lambda\right.$ ] they correspond to transitions with $\Delta \Lambda=2$; for example to the transition [411] $\frac{3}{2}^{+} \rightarrow[413] \frac{5}{2}^{+}$for the $K_{J}=\frac{3}{2} \rightarrow \frac{5}{2}$ interband transition in the $\mathrm{Eu}$ isotopes. In terms of the pseudo oscillator quantum numbers $\tilde{N}, \tilde{n}_{z}, \tilde{\Lambda}$ these interband transitions are characteristic of transitions from one member of a pseudo spinorbit doublet to the other, $\left[\tilde{N} \tilde{n}_{z} \tilde{\Lambda}\right] \Omega=\tilde{\Lambda}-\frac{1}{2} \rightarrow\left[\tilde{N} \tilde{n}_{z} \tilde{\Lambda}\right] \Omega^{\prime}=\tilde{\Lambda}+\frac{1}{2}$; e.g. $[\tilde{3} \tilde{1} \tilde{2}]^{\frac{3}{2}}{ }^{+} \rightarrow$ $[\tilde{3} \tilde{1} \tilde{2}]_{\frac{5}{2}}{ }^{+}$in the case of the Eu isotopes. The Ml hindrance factors for such transitions have been calculated in the Bohr-Mottelson rotational model under the assumption that the asymptotic pseudo oscillator quantum numbers $\tilde{N}, \tilde{n}_{z}, \tilde{\Lambda}$ are rigorously good quantum numbers. Results are given in table 14 of the appendix. In many cases these M1 hindrance factors are again much smaller than the experimentally observed ones; and we might conclude that a small amount of excitation out of the $1 \mathrm{~g}_{\frac{2}{2}}$ proton orbit, for odd $-Z$ nuclei, is therefore vital to a complete description of the large interband M1 hindrance factors. Such excitations have of course been excluded in both the asymptotic limit $\left[\tilde{N} \tilde{n}_{z} \tilde{\Lambda}\right]$ and in the pseudo SU(3) model. In this connection it is also interesting to note that the $\frac{7}{2}^{+}$first excited state in ${ }^{153} \mathrm{Eu}$ is the one state for which the observed magnetic moment is in disagreement with the prediction of the pseudo SU(3) model. We might expect a $\frac{7}{2}^{+}$state to be particularly sensitive to a small amount of excitation out of the $\lg _{\frac{9}{2}}$ proton orbit through interaction with the $\lg _{\frac{7}{2}}$ part of the $\tilde{N}=3$ proton configuration.

\section{Concluding remarks}

The very simple model, based on the leading pseudo SU(3) representations of the natural-parity parts of the proton and neutron configurations of heavy deformed nuclei, has had considerable success in predicting the right order of the $K$-bands in the Eu and $\mathrm{Tm}$ isotopes, with rotational spectra in qualitative agreement with the experimentally observed spectra. In view of the apparent general success of the model in predicting magnetic moments of heavy deformed nuclei, it is somewhat difficult to understand the complete failure of the model to predict the very large M1 hindrance factors which are observed for certain interband transitions. Although admixture of lower pseudo SU(3) representations and a small amount of configuration mixing with the unnatural-parity parts of the overall configurations could well contribute to cancellations in off-diagonal M1 matrix elements without making major contributions to the magnetic moments, it is much more likely that a small amount of excitation out of the $1 \mathrm{~g}_{\frac{2}{2}}$ proton orbit for odd- $Z$ nuclei, for example, is vital for quantitative agreement with the experimentally observed M1 transition probabilities, and this point is under investigation. Such excitations, however, would take us out of the framework of the simple pseudo SU(3) model. Nevertheless, in view of the considerable successes of the extreme zeroth-order version of our model, we may conclude that the pseudo $\mathrm{SU}(3)$ model deserves further investigation. 


\section{Appendix}

THE ASYMPTOTIC LIMIT $\left[\tilde{N} \tilde{n}_{z} \tilde{\Lambda}\right]$

To test the approximate validity of the pseudo quantum numbers in the asymptotic limit $\left[\tilde{N} \tilde{n}_{z} \tilde{\Lambda}\right]$, a number of nuclear properties have been calculated for the unified (rotational) model under the assumption that the Nilsson states can be replaced by single-particle states for which the pseudo oscillator numbers $\left[\tilde{N} \tilde{n}_{z} \tilde{\Lambda}\right]$ are good quantum numbers, (implying very specific values for the Nilsson coefficients). To follow the development it is best to start with a unified-model wave function for an odd- $A$ nucleus in which the deformed field function for the last (odd) nucleon is expanded in the $j \Omega$ scheme:

$$
\left[\frac{2 I+1}{16 \pi^{2}}\right]^{\frac{1}{2}} \sum_{j} c_{j \Omega}^{[\beta]}\left\{D_{M K}^{I} \chi_{\Omega}^{j}-(-1)^{I+\frac{1}{2}+j-\frac{1}{2}} D_{M-K}^{I} \chi_{-\Omega}^{j}\right\},
$$

where $\chi_{\Omega}^{j}$ are single-particle spherical shell-model wave functions for the odd nucleon and $c_{j \Omega}^{[\beta]}$ are the Nilsson coefficients for the state $[\beta]$. The notation assumes that $\Omega>0$. The transformation to the pseudo coupling scheme for the single-particle function $\chi_{\Omega}^{j}$

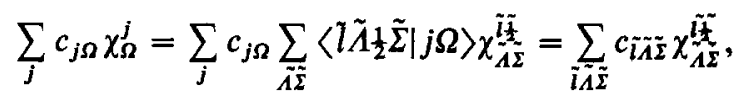

gives the deformed field coefficients $c_{i \tilde{I} \tilde{\Sigma}}$ in the pseudo scheme

$$
c_{\tilde{L} \tilde{\boldsymbol{\Sigma}} \tilde{\Sigma}}=\sum_{j} c_{j \Omega}\left\langle l \tilde{\Lambda} \tilde{\frac{1}{2}} \tilde{\Sigma} \mid j \Omega\right\rangle
$$

The Nilsson coefficient $c_{j \Omega}$ for maximum $j$, (e.g., $j=\frac{9}{2}$ for the $\lg _{\frac{3}{2}}$ orbit of the $N=4$ shell), is assumed to be zero. The remaining coefficients $c_{j \Omega}$ give the coefficients for the pseudo scheme, (e.g., the $c_{j \Omega}$ with $j=\frac{7}{2}$ and $\frac{5}{2}$ for $1 \mathrm{~g}_{\frac{3}{2}}$ and $2 \mathrm{~d}_{\frac{1}{2}}$ orbits determine the coefficients $c_{i \tilde{L} \tilde{\Sigma}}$ with $l=3$ ). The notation again assumes that $\tilde{A} \geqq 0$. The full wave function can then be put in the form

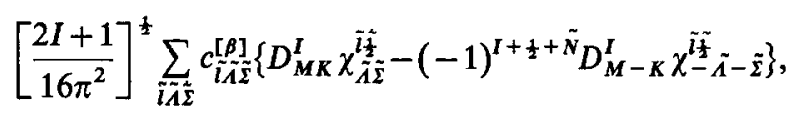

where we have used a symmetry property of the Clebsch-Gordan coefficient and the fact that $\tilde{N}-l=$ even for all $l$ of a pseudo oscillator shell $\tilde{N}$. If $\tilde{\Lambda}$ (and hence $\tilde{\Sigma}$ ) are assumed to be good quantum numbers, the sum in eq. (A.4) is over $l$ only. If $\bar{N} \tilde{n}_{z} \tilde{\Lambda}$ are all assumed to be good quantum numbers (the asymptotic limit), the coefficients $c_{i \tilde{S}_{\Sigma}}$ are simply the transformation coefficients between a cartesian and the spherical oscillator basis; see, e.g., eq. (2.3) of ref. ${ }^{32}$ ). Note that the $c_{\tilde{i} \boldsymbol{A}}^{\text {asympt. }}$ are independent of $\tilde{\Sigma}$. Starting with eq. (A.4), all calculations proceed exactly as calculations with Nilsson functions for the real oscillator basis expanded in $l-s$ coupling, where quantum numbers $N, l, \Lambda, \Sigma$ are replaced by $\tilde{N}, l, \tilde{\Lambda}, \tilde{\Sigma}$. In the asymptotic limit $\left[\tilde{N} \tilde{n}_{z} \tilde{\Lambda}\right]$, therefore, the matrix element of $\tilde{s}_{0}^{\prime}$, (the projection of $\tilde{s}$ along the body-fixed $z$-axis), for example, 
is simply $\tilde{\boldsymbol{\Sigma}}$. In the pseudo coupling scheme the magnetic moment operator

$$
\boldsymbol{\mu}=g_{R} \boldsymbol{I}+\left(g_{l}-g_{R}\right) \boldsymbol{j}+\left(g_{s}-g_{l}\right) s,
$$

is, (in the notation of eqs. (2a) and (5) of the text), given by

$$
\mu=g_{R} I+\left(g_{l}-g_{R}\right) \boldsymbol{j}-\frac{1}{3}\left(g_{s}-g_{l}\right) \tilde{s}+\left(g_{s}-g_{l}\right) \sum_{\tilde{l}^{\prime} l} \mu_{l^{\prime}} t\left(l^{\prime}, l\right)^{21,1},
$$

with

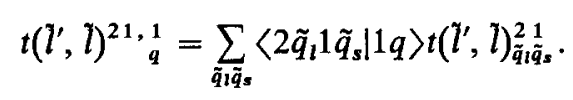

The matrix elements of $\mu$ between states of the type (A.4) can be determined by making use of the general expression $\left\langle\left[\tilde{N}^{\prime} \tilde{n}_{z}^{\prime} \tilde{\Lambda}^{\prime}\right] \Omega^{\prime} I^{\prime} K^{\prime} M^{\prime}\left|O_{q}^{k}\right|\left[\tilde{N} \tilde{n}_{z} \tilde{\Lambda}\right] \Omega I K M\right\rangle=\left[\frac{2 I+1}{2 I^{\prime}+1}\right]^{\frac{1}{2}}\left\langle I M k q \mid I^{\prime} M^{\prime}\right\rangle$

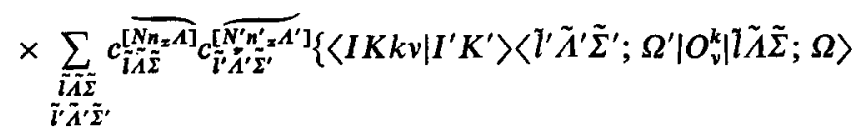

$$
\begin{aligned}
& \left.-(-1)^{I+\frac{1}{2}+\tilde{N}}\left\langle I K k v^{\prime} \mid I^{\prime}-K^{\prime}\right\rangle\left\langle I^{\prime}-\tilde{\Lambda}^{\prime}-\tilde{\Sigma}^{\prime} ;-\Omega^{\prime}\left|O_{v^{\prime}}^{k}\right| \tilde{I} \tilde{\Lambda} \tilde{\Sigma} ; \Omega\right\rangle\right\},
\end{aligned}
$$

where $O_{q}^{k}$ is a spherical tensor operator dependent on the degrees of freedom of the last odd nucleon only. In the asymptotic limit in which $\tilde{\Lambda}$ (and hence $\tilde{\Sigma}$ ) are good quantum numbers, we shall need the matrix element

$$
\begin{array}{r}
\left\langle\tilde{l}^{\prime} \tilde{\Lambda} \tilde{\Sigma} ; \Omega\left|t\left(\tilde{l}^{\prime}, \tilde{l}\right)^{21,}{ }_{0}^{1}\right| \tilde{l} \tilde{\Lambda} \tilde{\Sigma} ; \Omega\right\rangle=\langle 2010 \mid 10\rangle\left\langle l^{\prime} \tilde{\Lambda} l-\tilde{\Lambda} \mid 20\right\rangle\left\langle\frac{1}{2} \tilde{\Sigma} \frac{1}{2}-\tilde{\Sigma} \mid 10\right\rangle(-1)^{\tilde{N}+\tilde{l}-\tilde{A}+\frac{1}{2}-\tilde{\Sigma}} \\
=-\frac{\tilde{\Sigma}}{|\tilde{\Sigma}|} \frac{\left\langle l^{\prime} \tilde{\Lambda} 20 \mid \tilde{L}\right\rangle(-1)^{\tilde{N}}}{[2 \tilde{l}+1]^{\frac{1}{2}}} . \quad \text { (A.9) }
\end{array}
$$

For $\Omega=\frac{1}{2}$, we shall also need the matrix element

$$
\left\langle l^{\prime}-\tilde{\Lambda}-\tilde{\Sigma} ;-\frac{1}{2}\left|t\left(l^{\prime}, l\right)^{21,1}-1\right| l \tilde{\Lambda} \tilde{\Sigma} ;+\frac{1}{2}\right\rangle .
$$

For $\tilde{A}=0\left(\tilde{\Sigma}=\frac{1}{2}\right)$ this has the value

$$
\frac{\left\langle l^{\prime} 020 \mid \tilde{l} 0\right\rangle(-1)^{\tilde{N}}}{[2(2 l+1)]^{\frac{1}{2}}},
$$

and for $\tilde{\Lambda}=1\left(\tilde{\Sigma}=-\frac{1}{2}\right)$, it has the value

$$
-\left[\begin{array}{c}
3 \\
2 \tilde{l}+1
\end{array}\right]^{\frac{1}{t}}\left\langle\tilde{l}^{\prime} 12-2 \mid \tilde{l}-1\right\rangle(-1)^{\tilde{N}} \text {. }
$$

It is therefore convenient to define parameters $\alpha$ and $\beta$

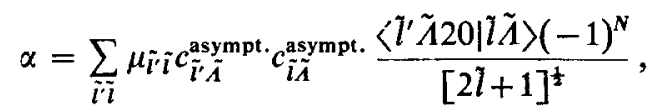

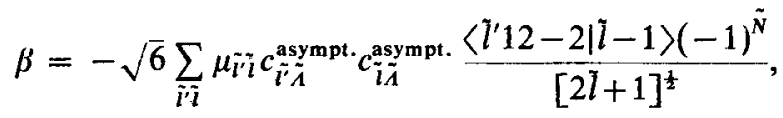


where the coefficients $\mu_{\eta^{\prime}}$ can be read from table 6 , and in the asymptotic limit of $\operatorname{good} \tilde{N} \tilde{n}_{z} \tilde{\Lambda}$ the expansion coefficients $c_{i \tilde{A}}$ follow from eq. (2.3) of ref. ${ }^{32}$ ). The magnetic moment for the ground state of rotational bands, $(I=K=\Omega)$, in the asymptotic limit $\left[\tilde{N} \tilde{n}_{z} \tilde{\Lambda}\right]$, then has the value

$$
\begin{aligned}
& \left\langle\left[\tilde{N} \tilde{n}_{z} \tilde{\Lambda}\right] K=\Omega=I|\mu|\left[\tilde{N} \tilde{n}_{z} \tilde{\Lambda}\right] K=\Omega=I\right\rangle \\
& \quad=g_{R} \frac{I}{I+1}+g_{l} \frac{I^{2}}{I+1}-(-1)^{I+\frac{1}{2}+\tilde{N}}\left(g_{l}-g_{R}\right) \delta_{K \frac{1}{4}} \delta_{\tilde{\Lambda} 0} \frac{I+\frac{1}{2}}{2(I+1)} \\
& \quad+\left(g_{s}-g_{l}\right)\left\{\frac{I}{2(I+1)} \frac{\tilde{\Sigma}}{|\tilde{\Sigma}|}\left(-\frac{1}{3}-2 \alpha\right)-(-1)^{I+\frac{1}{3}+\tilde{N}} \frac{I+\frac{1}{2}}{2(I+1)} \delta_{K !}\left[\left(\alpha-\frac{1}{3}\right) \delta_{\tilde{A} 0}+\beta \delta_{\tilde{A} 1}\right]\right\} .
\end{aligned}
$$

In the asymptotic limit $\left[N n_{z} \Lambda\right]$ for the real oscillator (Nilsson) scheme we get an analogous formula, if $\tilde{N}, \tilde{\Lambda}, \tilde{\Sigma}$ are replaced by $N, A, \Sigma ; \alpha$ and $\beta$ are set equal to zero, and the factor $-\frac{1}{3}$ in the $\left(g_{s}-g_{l}\right)$ term of eq. (A.13) is replaced by +1 . Results are given in tables 3 and 4 of the text.

Finally, it is interesting to calculate the $B(\mathrm{M} 1)$ value in the asymptotic limit $\left[\tilde{N} \tilde{n}_{z} \tilde{\Lambda}\right]$ for a transition from one member of a pseudo spin-orbit doublet to the other; that is, for the transition $\left[\tilde{N} \tilde{n}_{z} \tilde{\Lambda}\right] \Omega=\tilde{A}-\frac{1}{2} \rightarrow\left[\tilde{N} \tilde{n}_{z} \tilde{\Lambda}\right] \Omega=\tilde{A}+\frac{1}{2} \quad\left(I K=\tilde{A}-\frac{1}{2} \rightarrow I^{\prime} K^{\prime}=\right.$ $\left.\tilde{\Lambda}+\frac{1}{2}\right)$. For this purpose it is best to express the magnetic moment operator in the form

$$
\mu=g_{R} I+\left(g_{l}-g_{R}\right) \tilde{l}+\left(\frac{4}{3} g_{l}-\frac{1}{3} g_{s}-g_{R}\right) \tilde{s}+\left(g_{s}-g_{l}\right) \sum_{\tilde{l}^{\prime} t} \mu_{l^{\prime}} t\left(l^{\prime}, l^{21,1} .\right.
$$

In the limit of good $\tilde{N} \tilde{n}_{z} \tilde{\Lambda}$ this leads to the $B(\mathrm{M} 1)$ value

$$
\begin{gathered}
B(\mathrm{M} 1)\left(\left[\tilde{N} \tilde{n}_{z} \tilde{\Lambda}\right] \Omega=K=\tilde{\Lambda}-\frac{1}{2}, I \rightarrow\left[\tilde{N} \tilde{n}_{z} \tilde{\Lambda}\right] \Omega^{\prime}=K^{\prime}=\tilde{A}+\frac{1}{2}, I^{\prime}\right) \\
=\left(\frac{e h}{2 M c}\right)^{2} \frac{3}{8 \pi}\left[\left(\frac{4}{3} g_{l}-\frac{1}{3} g_{s}-g_{R}\right)+\alpha\left(g_{s}-g_{l}\right)\right]^{2}\left\langle I K 11 \mid I^{\prime} K+1\right\rangle^{2} .
\end{gathered}
$$

Since the same transition in the corresponding asymptotic limit $\left[N n_{z} \Lambda\right]$ is forbidden, $\Delta A=2$, (e.g., $[3 \overline{312}]]^{\frac{3}{2}}+\left[[\overline{312}]_{\frac{5}{2}}^{+} \text {corresponds to }[411]_{\frac{3}{2}}^{+} \rightarrow[413]\right]^{\frac{5}{2}}{ }^{+}$); it is useful to express the results in terms of Weisskopf hindrance factors $F_{\mathrm{W}}$, where

$$
F_{\mathrm{W}}=\frac{B(\mathrm{M} 1)_{\mathrm{W}}}{B(\mathrm{M} 1)} \text {, with } B(\mathrm{M} 1)_{\mathrm{W}}=\left(\frac{e \hbar}{2 M c}\right)^{2} \frac{3}{8 \pi} 15 .
$$

Hindrance factors, $F_{W}$, for the pseudo spin-orbit doublets of the $\tilde{N}=3$ and $\bar{N}=4$ shells are shown in table 14 for the transitions with $I=K=\tilde{A}-\frac{1}{2}$ and $I^{\prime}=K^{\prime}=$ $\tilde{A}+\frac{1}{2}$, (where we have assumed $g_{R}=0.4$ for the $\tilde{N}=3$ shell and $g_{R}=0.3$ for the $\tilde{N}=4$ shell). We note that for many of the pseudo spin-orbit doublets these hindrance factors are too small, when compared with the experimental values. 
TABLE 14

M1 hindrance factors $F_{\mathrm{w}}$ for transitions

\begin{tabular}{|c|c|c|c|}
\hline$\left[\tilde{N} \tilde{n}_{z} \tilde{A}\right]$ & $F_{\mathbf{w}}$ & {$\left[\tilde{N} \tilde{n}_{z} \tilde{A}\right]$} & $F_{\mathbf{w}}$ \\
\hline$[\tilde{30} \tilde{3}]$ & 1850 & {$[\tilde{40} \tilde{4}]$} & 6650 \\
\hline$[\tilde{3} \tilde{1} \tilde{2}]$ & 17 & {$[\tilde{4} \tilde{1} \tilde{3}]$} & 27 \\
\hline$[\tilde{3} \tilde{2} \tilde{1}]$ & 3.3 & $\tilde{4} \tilde{2} \tilde{2}]$ & 6.3 \\
\hline$[\tilde{30} \tilde{0} \tilde{1}]$ & 300 & {$[\tilde{4} \tilde{0} \tilde{2}]$} & $\$ 250$ \\
\hline & & $\tilde{[43 \tilde{1}} \tilde{1}]$ & 82 \\
\hline & & $\tilde{4 \pi \tilde{1}}]$ & 3.1 \\
\hline
\end{tabular}

\section{References}

1) J. P. Elliott, Proc. Roy. Soc. A245 (1958) 128, 562;

J. P. Elliott and M. Harvey, Proc. Roy. Soc. A272 (1963) 557

2) M. Harvey, Adv, in Nucl. Phys. vol. 1, (1968), ed. M. Baranger and E. Vogt

3) A. Arima, M. Harvey and K. Shimizu, Phys. Lett. 30B (1969) 517

4) K. T. Hecht and A. Adler, Nucl. Phys. A137 (1969) 129

5) R. Arvieu, Proc. Int. school of physics "Enrico Fermi", course XL, ed. M. Jean and R. A. Ricci (Academic Press, New York, 1969) 631

6) K. T. Hecht, Proc. 15th Solvay Conf. in physics, Brussels, 1970, to be published

7) W. P. Jones, L. W. Borgman, K. T. Hecht, J. Bardwick and W. C. Parkinson, Phys. Rev. C4 (1971) 580

8) F. T. Baker and R. Tickle, Phys. Rev. C5 (1972) 182

9) D. Strottman, Nucl. Phys. A188 (1972) 488

10) M. E. Bunker and C. W. Reich, Rev. Mod. Phys. 43 (1971) 348

11) B. R. Motteison and S. G. Nilsson, Mat. Fys. Skr. Dan. Vid. Selsk. 1, no. 8 (1959)

12) R. D. Ratna Raju, Dissertation, Univ, of Michigan, 1972

13) J. P. Draayer, to be published

14) J. P. Draaycr and Y. Akiyama, to be published

15) J. D. Vergados, Nucl. Phys. A111 (1968) 681

16) R. Arvieu and S. A. Moszkowski, Phys. Rev. 145 (1966) 830;

R. Arvieu, S. A. Moszkowski and A. Plastino, Phys. Rev. 145 (1966) 837

17) K. T. Hecht, J. B. McGrory and J. P. Draayer, Nucl. Phys. A197 (1972) 369

18) B. G. Wybourne, Symmetry principles and atomic spectroscopy (Wiley Interscience, 1970)

19) E. M. Ibrahim, Roy. Soc. Depository of mathematical tables (1950) no. 1

20) P. H. Butler and B. G. Wybourne, Atomic Data 3 (1971) 133

21) K. T. Hecht, Nucl. Phys. 62 (1965) 1

22) J. M. Eisenberg and W. Greiner, Nuclear theory, vol. 1 nuclear models (North-Holland, 1970)

23) J. de Boer and J. D. Rogers, Phys. Lett. 3 (1963) 304;

Z. Bochnacki and S. Ozaga, Nucl. Phys. 83 (1966) 619

24) G. H. Fuller and V. W. Cohen, Nucl. Data Tables A5 (1969) 433

25) J. Pluhar, Nucl. Phys. A167 (1971) 33;

J. P. Draayer, private communication

26) G. B. Holm, S. Borg and R. Rydberg, Conf. on the properties of nuclei far from the region of $\beta$-stability, Leysin, Switzerland, 1971;

B. H. Wildenthal, Phys. Rev. Lett. 22 (1969) 1118; Phys. Lett. 29B (1969) 274;

B. H. Wildenthal and D. C. Larson, Phys. Lett. 37B (1971) 266

27) J. Ungrin, D. G. Burke, M. W. Johns and W. P. Alford, Nucl. Phys. A132 (1969) 322

28) K. H. Bhatt and J. B. McGrory, Phys. Rev. C3 (1971) 2293

29) M. Richter, W. Henning and P. Kienle, Z. Phys. 218 (1969) 223

30) K. E. G. Löbner and S. G. Malmskog, Nucl. Phys. 80 (1966) 505

31) D. E. Raeside, J. J. Reidy and M. L. Wiedenbeck, Nucl. Phys. A114 (1968) 529

32) R. T. Sharp, H. C. yon Baeyer and S. C. Pieper, Nucl. Phys. A127 (1969) 513

33) A. S. Broad, Univ. of Michigan, private communication 